\title{
Unique, Yet Typical Oxyanion Holes in Aspartic Proteases
}

\author{
Mark Aldren M. Feliciano, Brian Gold
}

Department of Chemistry and Chemical Biology, University of New Mexico, Albuquerque, New Mexico 87131

\begin{tabular}{llc}
\hline \multicolumn{1}{c}{ Content } & Page \\
\hline \multicolumn{2}{c}{ Table of Contents } & S1 \\
\multicolumn{2}{c}{ Computational Details } & S2 \\
Figure S1 & Lewis structure of model system & S2 \\
Figure S2 & Lewis structure of extended model system & S3 \\
Figure S3 & Optimized structure of extended model system & S3 \\
Figure S4 & Charged Residues & S4 \\
Figure S5 & Optimized transition state structures & S4 \\
Figure S6 & Orbital depiction of anomeric interactions & S5 \\
Table S1 & Interaction energies & S5 \\
Table S2 & Aspartic Protease Inhibitors and Measured Parameters & S6 \\
Supplementary References & $\mathrm{S} 6$ \\
Coordinates Extracted from PDB Entry 3b80 & S7 \\
Optimized Coordinates & S9 \\
\hline
\end{tabular}


Computational Details: Coordinates were extracted from PDB entry 3b80, a gem-diol intermediate bound to HIV-1 protease. ${ }^{1}$ The model system is comprised of 153 atoms (including hydrogens), stemming from the entire DTG motif (D25, T26, G27, D25', T26', and G27'), the P1 and P1' residues of the substrate to the $\beta$-carbon of their side chains, along with a conserved water molecule residing between the substrate carbonyl oxygens ("flap water"), and the main chain amides of the flap. All termini were made into amides. Hydrogen atoms were added to extracted structures in idealized geometries with the program GaussView 6 (Wallingford, CT). Protonation was chosen in the gem-diol to match that determined by neutron diffraction, ${ }^{2}$ which guided the choice of species in previous and subsequent steps.

Partial relaxations were performed utilizing density functional theory. Specifically, we employed the M06-2X method $^{3}$ with the 6-31G(d) basis set as implemented with the Gaussian 16, rev. C.01 software. ${ }^{4}$ In each optimization, the aspartic acid side chains (D25/D25') and the glycine amides (G27/G27') of the DTG motif, the flap water, and all hydrogen atoms were allowed to relax (Figure S1). The substrate was allowed to completely relax, other than terminal carbon atoms. Frequency calculations confirmed the position on the potential energy surface-zero imaginary frequencies for minima and a single imaginary frequency for transition states. Localized orbitals interactions were obtained via Natural Bonding Orbital (NBO) analysis, utilizing the NBO 7.0 software. ${ }^{5}$ Interaction energies were obtained from the second-order perturbation analysis.

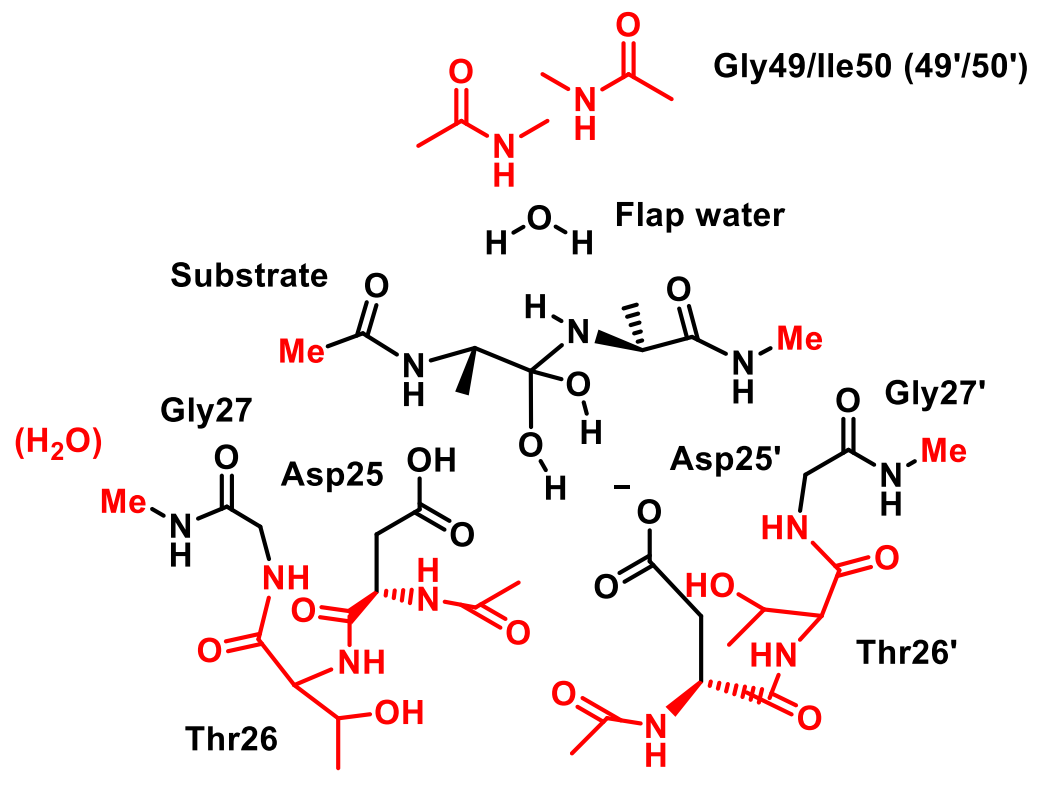

Figure S1. Lewis structure of model system extracted from PDB entry $3 \mathrm{~b} 80 .{ }^{1}$ Heavy atoms in red were frozen during geometry optimizations, which were carried out at the M062X/6-31G(d) level of theory. The crystallographic water molecule was included in optimized structures shown in Figures 4 and 5 of the main text. 


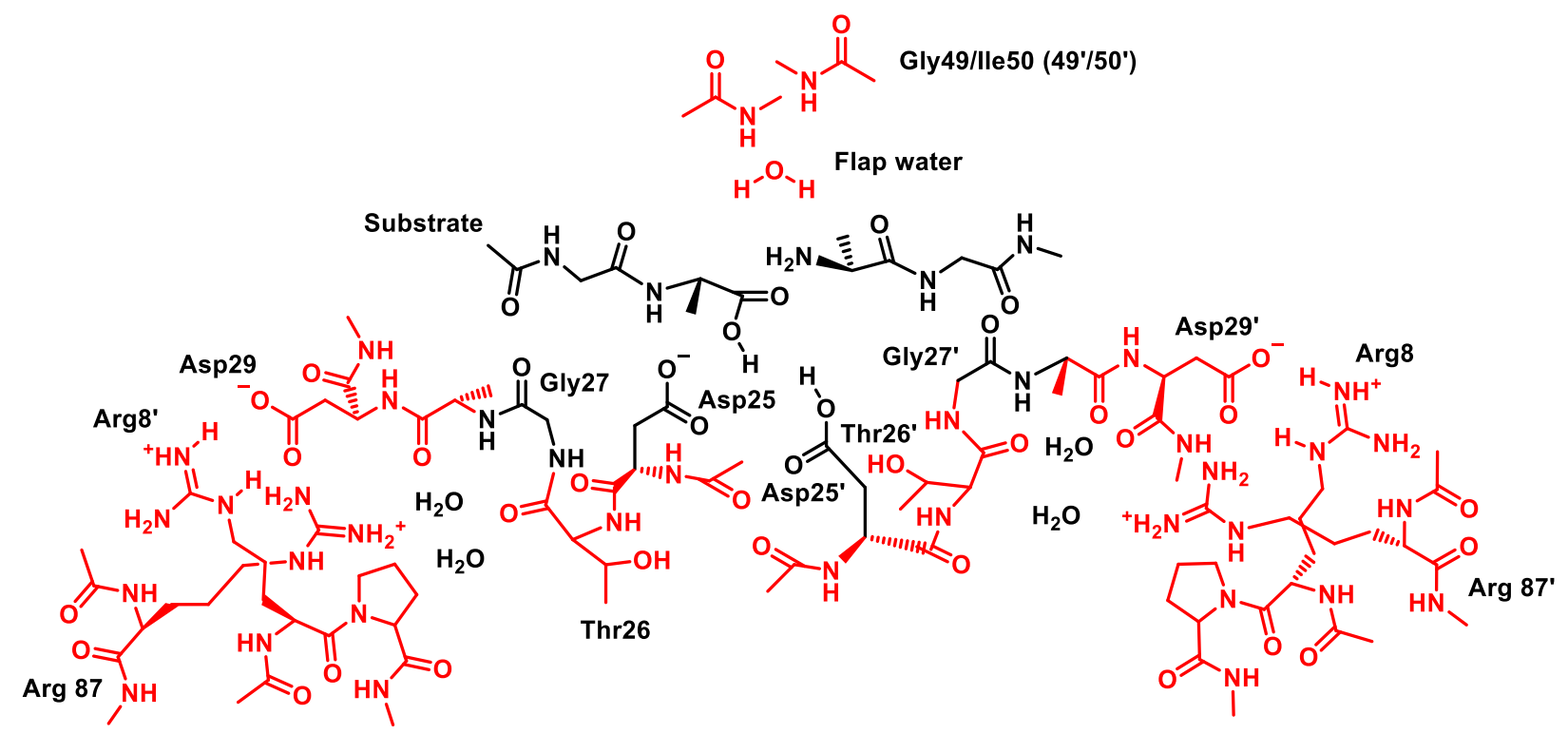

Figure S2. Lewis structure of the extended model system - including remote enzymic residues - extracted from PDB entry $3 \mathrm{~b} 80 .{ }^{1}$ Heavy atoms in red were frozen during geometry optimizations, which were carried out at the M062X/6$31 \mathrm{G}(\mathrm{d})$ level of theory. Optimized structures can be seen in Figure S3 and S4, and Figure 6 within the main text.

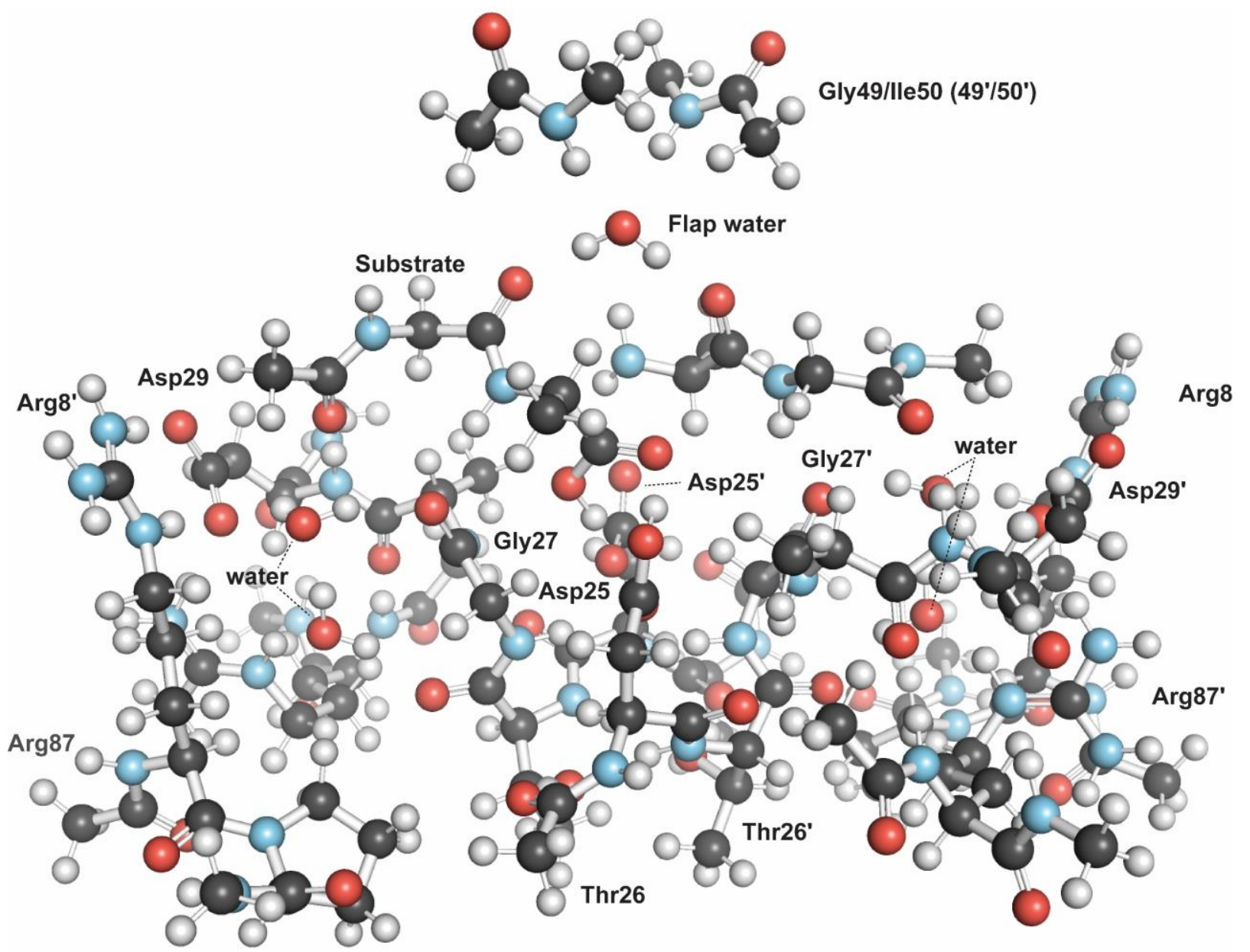

Figure S3. Optimized structure of the extended model system including remote enzymic residues (see Figure S2 for details). 


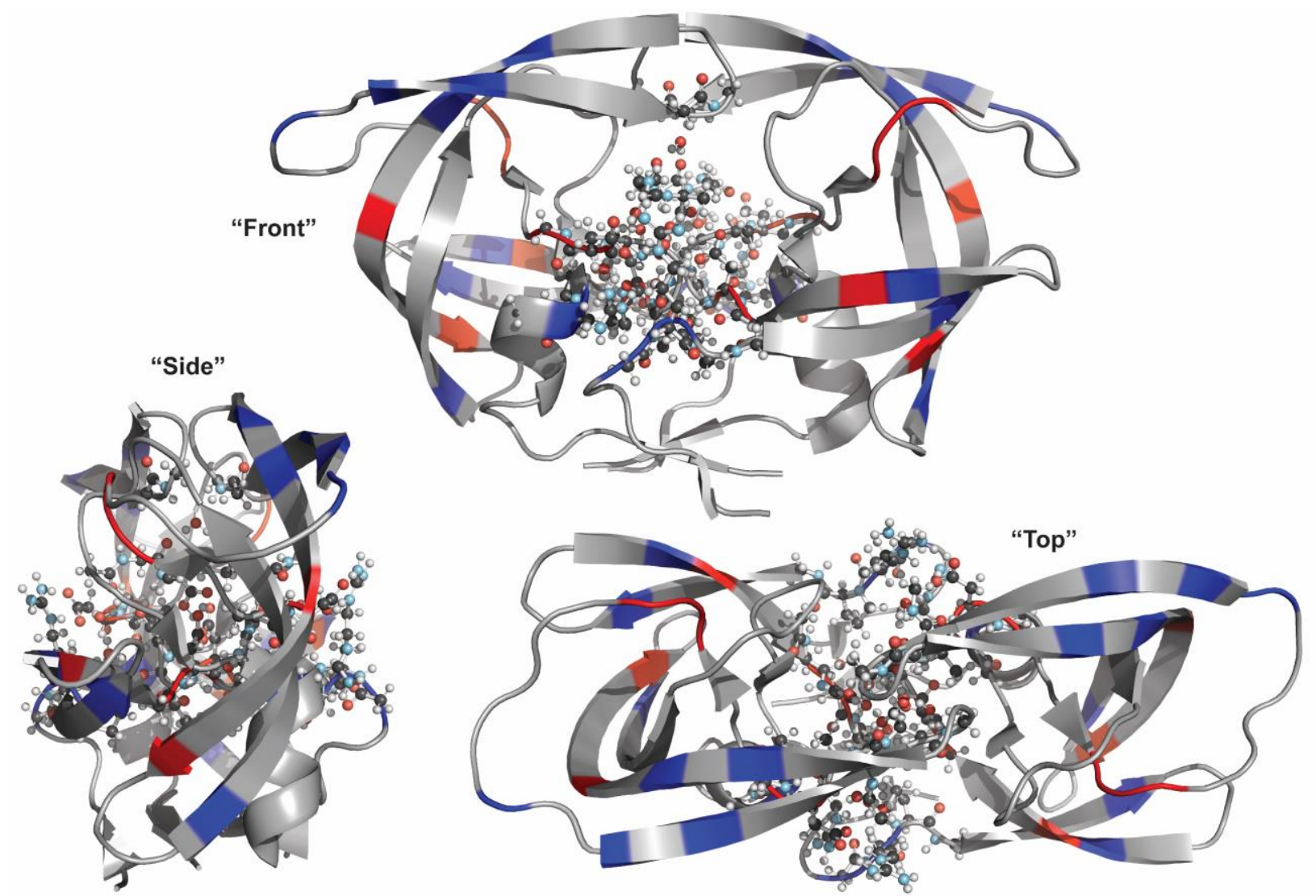

Figure S4. Overlay of the extended model system (Figure S2) and cartoon representation of PDB entry $3 b 80 .{ }^{1}$ Negatively charged residues are colored red; positively charged residues are blue.

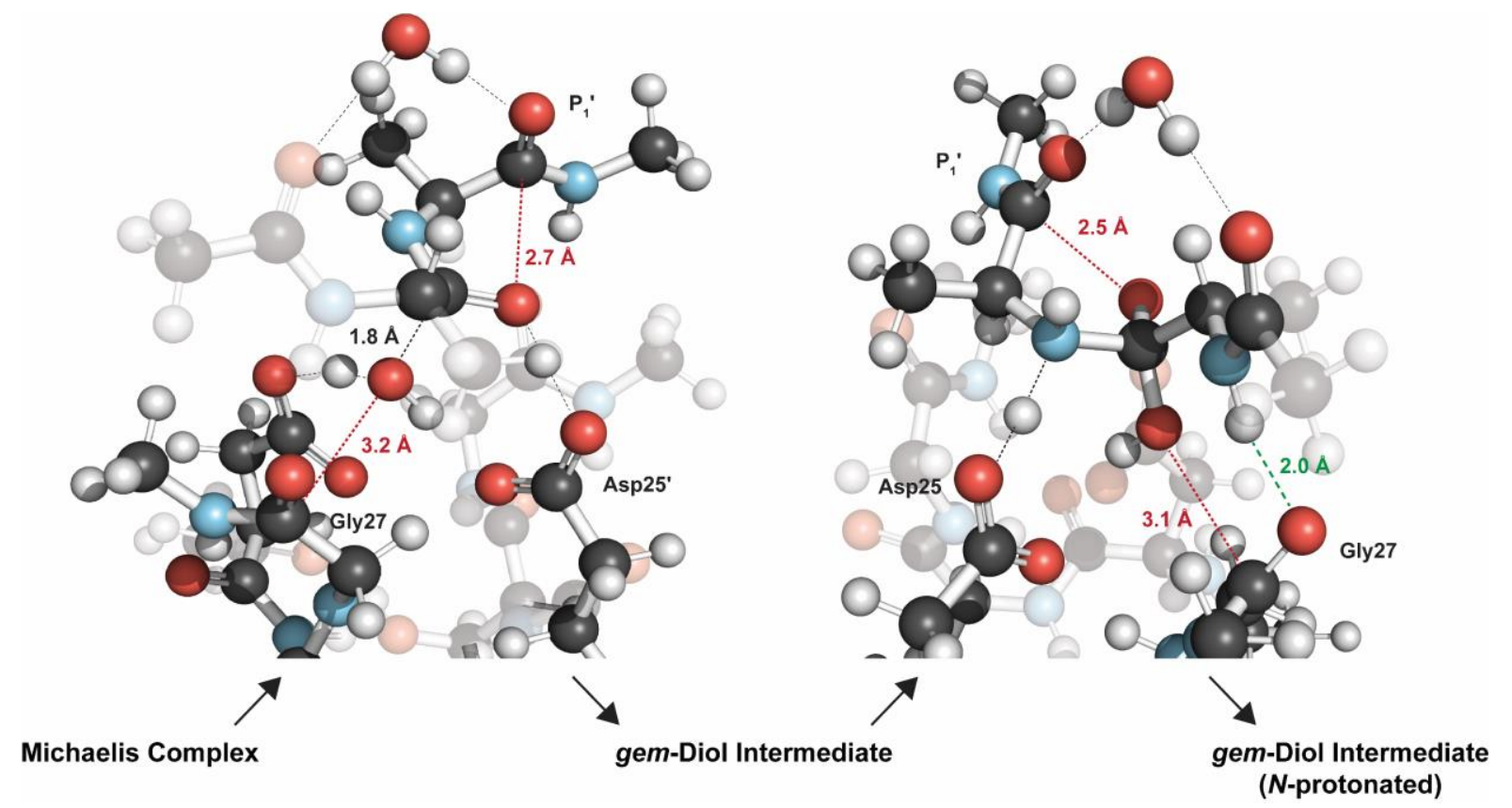

Figure S5. Optimized transition state structures from the Michaelis complex to gem-diol intermediate and proton transfer. For optimized structures of the Michaelis complex, product complex, and reactive intermediates, see Figure 3 of the main text. For the $\mathrm{C}-\mathrm{N}$ scission transition state, see Figures 4 and 5 of the main text. 


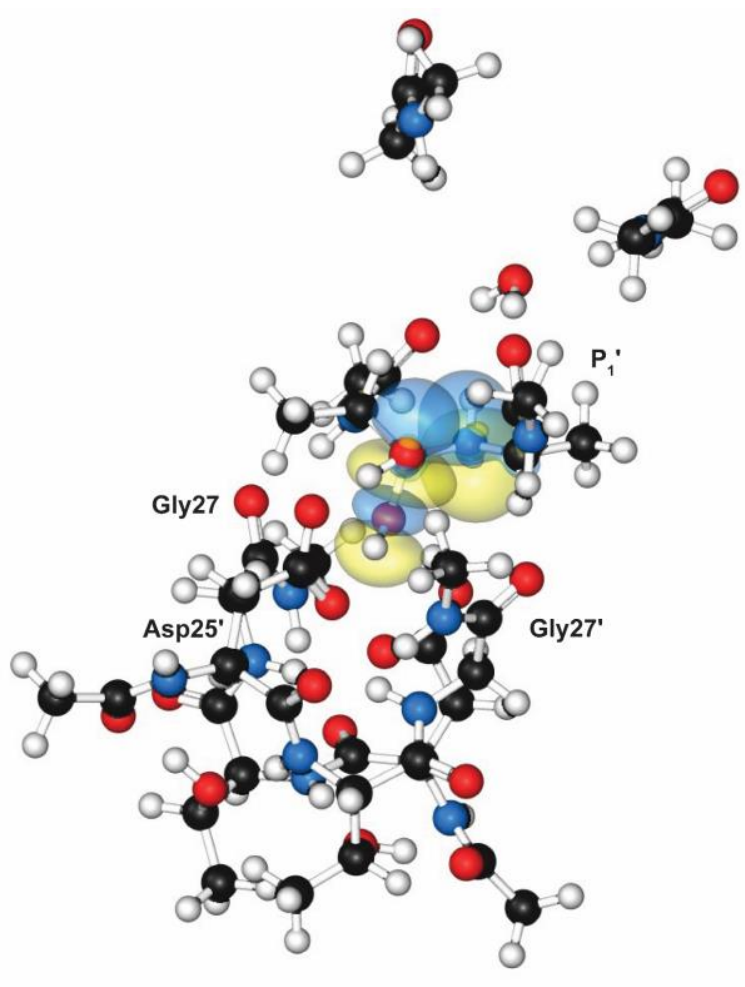

gem-Diol Intermediate

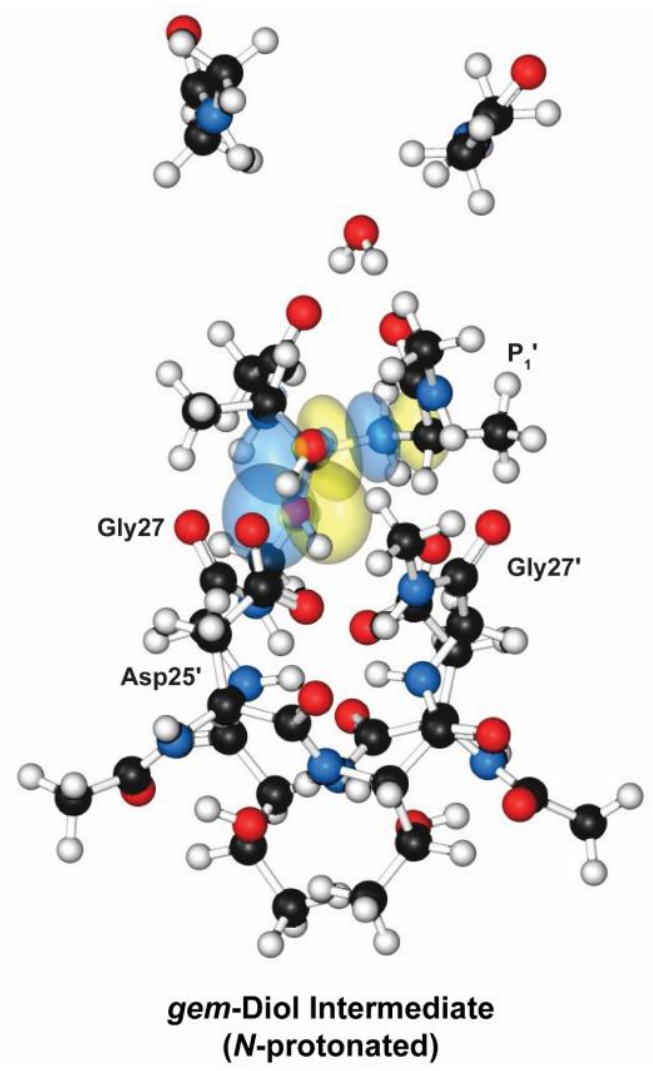

Figure S6. Anomeric interactions in calculated reactive intermediates. Depicted are $\mathrm{n}_{\mathrm{N}} \rightarrow \sigma^{*}{ }_{\mathrm{C}-\mathrm{O}}$ interactions in the gem-diol (left) and $\mathrm{n}_{\mathrm{O}} \rightarrow \sigma^{*}{ }_{\mathrm{C}-\mathrm{N}}$ interactions in the $N$-protonated gem-diol (right).

Table S1. Interaction Energies (kcal/mol) from $2^{\text {nd }}$ Order Perturbations in the NBO Analysis

\begin{tabular}{|c|c|c|c|c|c|}
\hline & \multirow{2}{*}{$\begin{array}{l}\begin{array}{l}\text { Intra-substrate } \\
\text { oxyanion hole }\end{array} \\
\mathrm{n}_{\mathrm{O}} \rightarrow \pi^{*}{ }_{\mathrm{C}=\mathrm{O}\left(\mathrm{P}^{\prime}\right)}\end{array}$} & \multirow{2}{*}{$\begin{array}{c}\text { Unique, yet typical } \\
\text { oxyanion hole }\end{array}$} & \multicolumn{3}{|c|}{$\begin{array}{l}\text { Amidic resonance } \rightarrow \text { anomeric interactions } \\
\qquad \rightarrow \text { acid resonance }\end{array}$} \\
\hline & & & $\mathrm{n}_{\mathrm{N}} \rightarrow \sigma^{*} \mathrm{C}-\mathrm{O}(\mathrm{LW})$ & $\mathrm{n}_{\mathrm{O}(\mathrm{LW})} \rightarrow \sigma^{*}{ }_{\mathrm{C}-\mathrm{N}}$ & $\mathrm{n}_{\mathrm{O}(\text { subst })} \rightarrow \sigma^{*} \mathrm{C}-\mathrm{N}$ \\
\hline $\begin{array}{l}\text { Michaelis } \\
\text { complex }\end{array}$ & $1.8^{a}$ & 1.0 & $98.6^{b}$ & - & 26.1 \\
\hline transition state 1 & 2.1 & $0.5^{a}$ & 70.5 & 1.0 & $6.6^{a}$ \\
\hline $\begin{array}{l}\text { gem-diol } \\
\text { intermediate }\end{array}$ & 4.7 & 0.2 & 13.1 & $7.6^{a}$ & $6.0^{a}$ \\
\hline transition state 2 & 6.7 & $0.5^{a}$ & 6.8 & $18.6^{a}$ & 20.0 \\
\hline $\begin{array}{l}N \text {-protonated } \\
\text { gem-diol }\end{array}$ & $5.1(5.2)^{c}$ & $0.5^{a}\left(0.7^{a}\right)^{c}$ & $0.9^{d}\left(0.9^{d}\right)^{c}$ & $26.5^{a}\left(26.2^{a}\right)^{c}$ & $19.9^{a}\left(20.5^{a}\right)^{c}$ \\
\hline transition state 3 & $1.9(1.9)$ & $0.4\left(0.8^{a}\right)^{c}$ & $0.1^{d}\left(0.1^{d}\right)^{c}$ & $72.6^{e}\left(71.4^{e}\right)^{c}$ & - \\
\hline product complex & $0.7^{a}\left(0.7^{a}\right)^{c}$ & $1.4^{a}\left(2.2^{a}\right)^{c}$ & - & $87.2^{e}\left(84.5^{e}\right)^{c}$ & - \\
\hline $\begin{array}{l}\text { extended product } \\
\text { complex }\end{array}$ & $0.6^{a}$ & $2.6^{a}$ & - & $88.2^{e}$ & - \\
\hline
\end{tabular}

${ }^{a}$ Sum of interactions from both lone pairs on oxygen; ${ }^{b}$ amidic resonance in the Michaelis complex; ${ }^{c}$ Values in parentheses are for optimizations including a constrained water molecule; ${ }^{d}$ Value given for $\sigma_{\mathrm{N}-\mathrm{H}} \rightarrow \sigma^{*} \mathrm{C}-\mathrm{O}(\mathrm{LW}) ;{ }^{e}$ Value given for carboxylic acid resonance $\mathrm{n}_{\mathrm{O}(\mathrm{LW})} \rightarrow \pi^{*} \mathrm{C}=\mathrm{O}$ (substrate) 
Table S2. Aspartic Protease Inhibitors and Measured Parameters

\begin{tabular}{|c|c|c|c|c|c|c|c|}
\hline $\begin{array}{l}\text { PDB } \\
\text { Entry }^{a}\end{array}$ & Resolution & Enzyme & Inhibitor & $d_{\mathrm{O} \cdots \mathrm{C}=\mathrm{O}},{ }^{b} \AA$ & $\theta,{ }^{c}$ deg. & $d_{\mathrm{O} \ldots \mathrm{O}},{ }^{d} \stackrel{\AA}{\mathrm{A}}$ & $d_{\mathrm{O} \cdots \mathrm{N}},{ }^{e} \AA$ \\
\hline 4hla & 1.95 & $\begin{array}{c}\text { HIV-1 } \\
\text { Protease }\end{array}$ & darunavir & 3.5 & 87.2 & 2.9 & 3.2 \\
\hline $6 \mathrm{pjb}$ & 1.98 & $\begin{array}{c}\text { HIV-1 } \\
\text { Protease }\end{array}$ & lopinavir & 3.8 & 89.1 & 2.9 & 3.9 \\
\hline 3 oxc & 1.16 & $\begin{array}{c}\text { HIV-1 } \\
\text { Protease }\end{array}$ & saquinavir & 3.5 & 95.6 & 2.6 & 3.7 \\
\hline 1hxw & 1.80 & $\begin{array}{c}\text { HIV-1 } \\
\text { Protease }\end{array}$ & ritonavir & 3.8 & 91.1 & 2.7 & 3.5 \\
\hline 3ekx & 1.97 & $\begin{array}{c}\text { HIV-1 } \\
\text { Protease }\end{array}$ & nelfinavir & 3.4 & 88.7 & 2.9 & 3.4 \\
\hline 3nu3 & 1.02 & $\begin{array}{c}\text { HIV-1 } \\
\text { Protease }\end{array}$ & amprenavir & 3.4 & 89.9 & 2.7 & 3.1 \\
\hline 3el1 & 1.70 & $\begin{array}{c}\text { HIV-1 } \\
\text { Protease }\end{array}$ & atazanavir & 3.2 & 80.0 & 2.8 & 3.3 \\
\hline $1 \mathrm{sdt}$ & 1.30 & $\begin{array}{c}\text { HIV-1 } \\
\text { Protease } \\
\end{array}$ & indinavir & 3.6 & 92.1 & 2.7 & 3.1 \\
\hline \multicolumn{4}{|c|}{ Average } & $3.5 \pm 0.2$ & $89.2 \pm 4.2$ & $2.8 \pm 0.1$ & $3.4 \pm 0.3$ \\
\hline 6dif & 1.20 & $\begin{array}{c}\text { HIV-1 } \\
\text { Protease }\end{array}$ & tipranavir & 4.1 & 95.7 & 4.1 & - \\
\hline $5 \mathrm{hvp}$ & 2.00 & $\begin{array}{c}\text { HIV-1 } \\
\text { Protease }\end{array}$ & pepstatin & 3.6 & 80.0 & 3.0 & 3.0 \\
\hline $2 \mathrm{v} 0 \mathrm{z}^{f}$ & 2.20 & renin & aliskiren & $3.1 / 3.1$ & $90.1 / 87.7$ & $2.8 / 2.9$ & $2.9 / 3.0$ \\
\hline $2 \mathrm{p} 4 \mathrm{j}^{f}$ & 2.50 & $\begin{array}{l}\text { Memapsin } 2 \\
(\beta \text {-secretase) }\end{array}$ & $\begin{array}{c}23 \mathrm{I} \\
\text { (PDB ID) }\end{array}$ & $\begin{array}{l}3.6 \\
3.5 \\
3.6 \\
3.7\end{array}$ & $\begin{array}{l}97.6 \\
89.3 \\
90.1 \\
95.7\end{array}$ & $\begin{array}{l}3.0^{g} \\
3.2^{g} \\
3.1^{g} \\
3.2^{g}\end{array}$ & $\begin{array}{l}3.5 \\
3.4 \\
3.5 \\
3.7\end{array}$ \\
\hline
\end{tabular}

${ }^{a}$ See reference $6 ;{ }^{b}$ Substrate-Gly27 $\mathrm{n}_{\mathrm{O}} \rightarrow \pi^{*} \mathrm{C}=\mathrm{O}$ donor-acceptor distance; ${ }^{c}$ Bürgi-Dunitz trajectory; ${ }^{d}$ Gly27 oxygenstructural water; ${ }^{e} \mathrm{Gly} 27$ oxygen-substrate nitrogen; ${ }^{f}$ Multiple bound conformers within the unit cell; ${ }^{g}$ Gly 27 oxygenisophthalamide nitrogen

\section{Supplementary References}

1. Kovalevsky, A. Y.; Chumanevich, A. A.; Liu, F.; Louis, J. M.; Weber, I. T. Caught in the Act: The $1.5 \AA$ resolution crystal structures of the HIV-1 protease and the I54V mutant reveal a tetrahedral reaction intermediate. Biochemistry 2007, 46, 1485414864.

2. Coates, L.; Tuan, H.-F.; Tomanicek, S.; Kovalevsky, A.; Mustyakimov, M.; Erskine, P.; Cooper, J. The catalytic mechanism of an aspartic proteinase explored with neutron and X-ray diffraction. J. Am. Chem. Soc. 2008, 130, 7235-7237.

3. Zhao, Y.; Truhlar, D.G. The M06 suite of density functionals for main group thermochemistry, thermochemical kinetics, noncovalent interactions, excited states, and transition elements: Two new functionals and systematic testing of four M06-class functionals and 12 other functionals. Theor. Chem. Account 2008, 120, 215-241.

4. Frisch, M. J.; Trucks, G. W.; Schlegel, H. B.; Scuseria, G. E.; Robb, M. A.; Cheeseman, J. R.; Scalmani, G.; Barone, V.; Petersson, G. A.; Nakatsuji, H.; Li, X.; Caricato, M.; Marenich, A. V.; Bloino, J.; Janesko, B. G.; Gomperts, R.; Mennucci, B.; Hratchian, H. P.; Ortiz, J. V.; Izmaylov, A. F.; Sonnenberg, J. L.; Williams-Young, D.; Ding, F.; Lipparini, F.; Egidi, F.; Goings, J.; Peng, B.; Petrone, A.; Henderson, T.; Ranasinghe, D.; Zakrzewski, V. G.; Gao, J.; Rega, N.; Zheng, G.; Liang, W.; Hada, M.; Ehara, M.; Toyota, K.; Fukuda, R.; Hasegawa, J.; Ishida, M.; Nakajima, T.; Honda, Y.; Kitao, O.; Nakai, H.; Vreven, T.; Throssell, K.; Montgomery, J. A., Jr.; Peralta, J. E.; Ogliaro, F.; Bearpark, M. J.; Heyd, J. J.; Brothers, E. N.; Kudin, K. N.; Staroverov, V. N.; Keith, T. A.; Kobayashi, R.; Normand, J.; Raghavachari, K.; Rendell, A. P.; Burant, J. C.; Iyengar, S. S.; 
Tomasi, J.; Cossi, M.; Millam, J. M.; Klene, M.; Adamo, C.; Cammi, R.; Ochterski, J. W.; Martin, R. L.; Morokuma, K.; Farkas, O.; Foresman, J. B.; Fox, D. J. Gaussian 16, Revision C.01; Gaussian, Inc.: Wallingford, CT, 2016.

5. Glendening, E. D.; Badenhoop, J. K.; Reed, A. E.; Carpenter, J. E.; Bohmann, J. A.; Morales, C. M.; Karafiloglou, P.; Landis, C. R.; Weinhold, F. NBO 7.0; Theoretical Chemistry Institute, University of Wisconsin-Madison: Madison, WI, 2018.

6. (a) Fitzgerald, P. M.; McKeever, B. M.; VanMiddlesworth, J. F.; Springer, J. P.; Heimbach, J. C.; Leu, C. T.; Herber, W. K.; Dixon, R. A.; Darke, P. L. Crystallographic analysis of a complex between human immunodeficiency virus type 1 protease and acetyl-pepstatin at 2.0-Å resolution. J. Biol. Chem. 1990, 265, 14209-14219.; (b) Yedidi, R. S.; Maeda, K.; Fyvie, W. S.; Steffey, M.; Davis, D. A.; Palmer, I.; Aoki, M.; Kaufman, J. D.; Stahl, S. J.; Garimella, H.; Das, D.; Wingfield, P. T.; Ghosh, A. K.; Mitsuya, H. P2' benzene carboxylic acid moiety is associated with decrease in cellular uptake: Evaluation of novel nonpeptidic HIV-1 protease inhibitors containing P2 bis-tetrahydrofuran moiety. Antimicrob. Agents Chemother. 2013, 57, 4920-4927.; (c) Rusere, L. N.; Lockbaum, G. J.; Henes, M.; Lee, S.-K.; Spielvogel, E.; Rao, D. N.; Kosovrasti, K.; Nalivaika, E. A.; Swanstrom, R.; Kurt Yilmaz, N.; Schiffer, C. A.; Ali, A. Structural analysis of potent hybrid HIV-1 protease inhibitors containing bis-tetrahydrofuran in a pseudosymmetric dipeptide isostere. J. Med. Chem. 2020, 63, 8296-8313.; (d) Tie, Y.; Kovalevsky; A.Y., Boross, P.; Wang, Y.-F.; Ghosh, A.K.; Tozser, J.; Harrison, R.W.; Weber, I.T. Atomic resolution crystal structures of HIV-1 protease and mutants V82A and I84V with saquinavir. Proteins 2007, 67, 232-242.; (e) Kempf, D. J.; Marsh, K. C.; Denissen, J. F.; McDonald, E.; Vasavanonda, S.; Flentge, C. A.; Green, B. E.; Fino, L.; Park, C. H.; Kong, X. P. ABT-538 is a potent inhibitor of human immunodeficiency virus protease and has high oral bioavailability in humans. Proc. Natl. Acad. Sci. U. S. A. 1995, 92, 2484-2488.; (f) King, N. M.; Prabu-Jeyabalan, M.; Bandaranayake, R. M.; Nalam, M. N. L.; Nalivaika, E. A.; Özen, A.; Haliloğlu, T.; Yılmaz, N. K.; Schiffer, C. A. Extreme entropy-enthalpy compensation in a drug-resistant variant of HIV-1 protease ACS Chem. Biol. 2012, 7, 1536-1546.; (g) Shen, C.-H.; Wang, Y.-F.; Kovalevsky, A. Y.; Harrison, R. W.; Weber, I. T. Amprenavir complexes with HIV-1 protease and its drug-resistant mutants altering hydrophobic clusters. FEBS J. 2010, 277, 3699-3714.; (h) Mahalingam, B.; Wang, Y.-F.; Boross, P. I.; Tozser, J.; Louis, J. M.; Harrison, R. W.; Weber, I. T. Crystal structures of HIV protease V82A and L90M mutants reveal changes in the indinavirbinding site. Eur. J. Biochem. 2004, 271, 1516-1524.; (i) Rahuel, J.; Rasetti, V.; Maibaum, J.; Rüeger, H.; Göschke, R.; Cohen, N. C.; Stutz, S.; Cumin, F.; Fuhrer, W.; Wood, J. M.; Grütter, M. G. Structure-based drug design: The discovery of novel nonpeptide orally active inhibitors of human renin. Chem. Biol. 2000, 7, 493-504.; (j) Ghosh, A. K.; Kumaragurubaran, N.; Hong, L.; Kulkarni, S. S.; Xu, X.; Chang, W.; Weerasena, V.; Turner, R.; Koelsch, G.; Bilcer, G.; Tang, J. Design, synthesis, and X-ray structure of potent memapsin 2 ( $\beta$-secretase) inhibitors with isophthalamide derivatives as the P2-P3ligands. J. Med. Chem. 2007, 50, 2399-2407.

\begin{tabular}{|c|c|c|c|}
\hline & $\begin{array}{l}\text { tracted F } \\
\text { del in Fig }\end{array}$ & $\begin{array}{l}\text { m PDB } \\
\text { S1 }\end{array}$ & try $3680^{1}$ \\
\hline${ }_{C}$ & 9.08400000 & 30.75900000 & 15.47400000 \\
\hline C & 9.82300000 & 30.06600000 & 16.60400000 \\
\hline 0 & 9.21100000 & 29.44700000 & 17.47700000 \\
\hline $\mathrm{N}$ & 11.16000000 & 30.14700000 & 16.62300000 \\
\hline $\mathrm{C}$ & 11.95400000 & 29.24200000 & 17.43300000 \\
\hline C & 12.87700000 & 29.98700000 & 18.38200000 \\
\hline 0 & 13.96000000 & 30.41300000 & 17.98500000 \\
\hline $\mathrm{C}$ & 12.74800000 & 28.36500000 & 16.48800000 \\
\hline $\mathrm{C}$ & 13.47500000 & 27.25200000 & 17.21200000 \\
\hline 0 & 13.58300000 & 27.30100000 & 18.46900000 \\
\hline 0 & 13.95100000 & 26.30200000 & 16.50900000 \\
\hline $\mathrm{N}$ & 12.43300000 & 30.13900000 & 19.64300000 \\
\hline $\mathrm{C}$ & 13.23200000 & 30.85700000 & 20.60600000 \\
\hline C & 14.52100000 & 30.08300000 & 20.94600000 \\
\hline 0 & 15.45500000 & 30.68000000 & 21.49800000 \\
\hline $\mathrm{C}$ & 12.45100000 & 31.14000000 & 21.91200000 \\
\hline C & 11.21300000 & 32.00100000 & 21.61300000 \\
\hline 0 & 11.99900000 & 29.92000000 & 22.46100000 \\
\hline $\mathrm{N}$ & 14.60100000 & 28.80300000 & 20.64300000 \\
\hline C & 15.78000000 & 27.99700000 & 20.93900000 \\
\hline C & 16.86900000 & 28.10500000 & 19.88900000 \\
\hline 0 & 17.95900000 & 27.56400000 & 20.08400000 \\
\hline $\mathrm{N}$ & 16.56500000 & 28.79700000 & 18.79500000 \\
\hline C & 17.49500000 & 28.92700000 & 17.69300000 \\
\hline $\mathrm{C}$ & 21.88000000 & 20.59300000 & 17.36500000 \\
\hline C & 21.90900000 & 19.12500000 & 17.70400000 \\
\hline 0 & 22.85900000 & 18.38500000 & 17.47800000 \\
\hline $\mathrm{N}$ & 20.77600000 & 18.75000000 & 18.27300000 \\
\hline C & 20.57700000 & 17.34400000 & 18.42300000 \\
\hline C & 12.99700000 & 27.99100000 & 26.79400000 \\
\hline C & 12.90800000 & 27.95900000 & 25.28300000 \\
\hline 0 & 13.28400000 & 28.93400000 & 24.63500000 \\
\hline $\mathrm{N}$ & 12.42000000 & 26.85900000 & 24.71900000 \\
\hline C & 12.51700000 & 26.60500000 & 23.28900000 \\
\hline C & 11.17400000 & 26.37300000 & 22.61700000 \\
\hline 0 & 10.62100000 & 25.24500000 & 22.64500000 \\
\hline C & 13.40200000 & 25.37800000 & 23.07600000 \\
\hline C & 13.80200000 & 25.12400000 & 21.62700000 \\
\hline 0 & 13.21300000 & 25.79000000 & 20.73500000 \\
\hline 0 & 14.70500000 & 24.25100000 & 21.45800000 \\
\hline $\mathrm{N}$ & 10.60500000 & 27.41800000 & 21.99600000 \\
\hline C & 9.32800000 & 27.23200000 & 21.31600000 \\
\hline C & 9.41300000 & 26.35400000 & 20.05700000 \\
\hline 0 & 8.36200000 & 25.91200000 & 19.54700000 \\
\hline C & 8.72600000 & 28.59800000 & 20.91700000 \\
\hline
\end{tabular}

\begin{tabular}{|c|c|c|c|}
\hline $\mathrm{C}$ & 8.50900000 & 29.44100000 & 22.16400000 \\
\hline 0 & 9.65000000 & 29.26800000 & 20.05500000 \\
\hline $\mathrm{N}$ & 10.63400000 & 26.10500000 & 19.56600000 \\
\hline C & 10.88600000 & 25.22800000 & 18.43800000 \\
\hline $\mathrm{C}$ & 10.96700000 & 23.74300000 & 18.76500000 \\
\hline 0 & 11.13600000 & 22.88000000 & 17.89900000 \\
\hline $\mathrm{N}$ & 10.84500000 & 23.40200000 & 20.04800000 \\
\hline C & 10.93400000 & 22.05000000 & 20.52400000 \\
\hline C & 16.72600000 & 18.17800000 & 14.88100000 \\
\hline C & 17.44600000 & 18.34500000 & 13.54200000 \\
\hline 0 & 17.43600000 & 17.45600000 & 12.69600000 \\
\hline $\mathrm{N}$ & 18.05000000 & 19.49400000 & 13.40100000 \\
\hline C & 18.68700000 & 19.84400000 & 12.13900000 \\
\hline C & 19.69500000 & 25.74500000 & 16.87400000 \\
\hline $\mathrm{C}$ & 18.77900000 & 24.59700000 & 17.29600000 \\
\hline 0 & 18.90400000 & 23.48400000 & 16.76600000 \\
\hline $\mathrm{C}$ & 17.17100000 & 23.82600000 & 18.99200000 \\
\hline C & 14.57700000 & 21.02200000 & 17.44400000 \\
\hline 0 & 15.62200000 & 20.38300000 & 17.49600000 \\
\hline $\mathrm{N}$ & 17.86500000 & 24.88900000 & 18.25400000 \\
\hline $\mathrm{C}$ & 15.69100000 & 23.70200000 & 18.53600000 \\
\hline C & 17.37100000 & 24.09100000 & 20.50000000 \\
\hline 0 & 15.11900000 & 22.83700000 & 19.49400000 \\
\hline 0 & 14.96900000 & 24.93600000 & 18.63500000 \\
\hline $\mathrm{N}$ & 15.60900000 & 23.24900000 & 17.13800000 \\
\hline $\mathrm{C}$ & 14.44300000 & 22.43200000 & 16.81300000 \\
\hline C & 14.11600000 & 22.15400000 & 15.30500000 \\
\hline $\mathrm{N}$ & 13.36100000 & 20.61100000 & 17.92900000 \\
\hline C & 13.25100000 & 19.14500000 & 18.05300000 \\
\hline 0 & 18.02500000 & 20.99900000 & 16.51400000 \\
\hline \multirow{2}{*}{\multicolumn{4}{|c|}{$\begin{array}{l}\text { Coordinates Extracted From PDB Entry } 3 \mathrm{bs0}^{1} \text { to } \\
\text { generate the model in Figure } \mathrm{S} 2\end{array}$}} \\
\hline & & & \\
\hline C & 0.74800000 & 23.52900000 & 18.59300000 \\
\hline C & 2.05300000 & 24.22000000 & 18.21900000 \\
\hline 0 & 2.40800000 & 25.21200000 & 18.82800000 \\
\hline $\mathrm{N}$ & 2.79200000 & 23.72900000 & 17.23000000 \\
\hline C & 3.97000000 & 24.40500000 & 16.73500000 \\
\hline C & 3.58400000 & 25.81800000 & 16.30200000 \\
\hline 0 & 2.60500000 & 25.93000000 & 15.56000000 \\
\hline C & 4.58000000 & 23.62800000 & 15.58900000 \\
\hline C & 5.10900000 & 22.24100000 & 15.96200000 \\
\hline C & 5.87300000 & 21.56700000 & 14.81600000 \\
\hline $\mathrm{N}$ & 6.28100000 & 20.23900000 & 15.22800000 \\
\hline C & 6.54300000 & 19.20600000 & 14.45200000 \\
\hline $\mathrm{N}$ & 6.44400000 & 19.31300000 & 13.13700000 \\
\hline $\mathrm{N}$ & 6.90800000 & 18.04600000 & 14.97600000 \\
\hline
\end{tabular}




\begin{tabular}{|c|c|c|c|}
\hline $\mathrm{N}$ & 4.33500000 & 26.82200000 & 16.76000000 \\
\hline $\mathrm{C}$ & 4.05900000 & 28.21000000 & 16.32800000 \\
\hline $\mathrm{C}$ & 4.58800000 & 28.54700000 & 14.93100000 \\
\hline 0 & 5.62000000 & 29.19600000 & 14.68200000 \\
\hline C & 4.80300000 & 29.01300000 & 17.41500000 \\
\hline C & 5.96600000 & 28.15100000 & 17.78900000 \\
\hline C & 5.46800000 & 26.72000000 & 17.69600000 \\
\hline $\mathrm{N}$ & 8.46200000 & 29.78800000 & 14.57000000 \\
\hline $\mathrm{C}$ & 9.08400000 & 30.75900000 & 15.47400000 \\
\hline C & 9.82300000 & 30.06600000 & 16.60400000 \\
\hline 0 & 9.21100000 & 29.44700000 & 17.47700000 \\
\hline C & 7.99071267 & 31.66607690 & 16.06858752 \\
\hline $\mathrm{N}$ & 16.56500000 & 28.79700000 & 18.79500000 \\
\hline $\mathrm{C}$ & 17.49500000 & 28.92700000 & 17.69300000 \\
\hline C & 18.19100000 & 30.27800000 & 17.70600000 \\
\hline 0 & 17.53700000 & 31.31700000 & 17.71700000 \\
\hline $\mathrm{C}$ & 16.80200000 & 28.78100000 & 16.35500000 \\
\hline $\mathrm{N}$ & 20.77600000 & 18.75000000 & 18.27300000 \\
\hline $\mathrm{C}$ & 20.57700000 & 17.34400000 & 18.42300000 \\
\hline $\mathrm{C}$ & 20.18600000 & 36.72900000 & 24.59500000 \\
\hline C & 19.42400000 & 35.40800000 & 24.57200000 \\
\hline 0 & 18.17800000 & 35.43900000 & 24.44000000 \\
\hline $\mathrm{N}$ & 20.12200000 & 34.27300000 & 24.69500000 \\
\hline $\mathrm{C}$ & 19.36100000 & 33.03200000 & 24.79900000 \\
\hline C & 18.44500000 & 33.07300000 & 26.00900000 \\
\hline 0 & 18.89100000 & 33.50600000 & 27.09100000 \\
\hline $\mathrm{C}$ & 20.30800000 & 31.85800000 & 24.89300000 \\
\hline $\mathrm{C}$ & 21.12900000 & 31.57500000 & 23.65100000 \\
\hline C & 22.00500000 & 30.34100000 & 23.89000000 \\
\hline $\mathrm{N}$ & 22.82300000 & 30.14200000 & 22.69100000 \\
\hline $\mathrm{C}$ & 23.96900000 & 29.46600000 & 22.63000000 \\
\hline $\mathrm{N}$ & 24.45000000 & 28.90400000 & 23.73500000 \\
\hline $\mathrm{N}$ & 24.58600000 & 29.38300000 & 21.45600000 \\
\hline $\mathrm{N}$ & 17.19700000 & 32.64300000 & 25.86300000 \\
\hline $\mathrm{C}$ & 16.30000000 & 32.62000000 & 27.03000000 \\
\hline C & 16.54200000 & 31.45700000 & 28.00000000 \\
\hline 0 & 15.79400000 & 30.48000000 & 28.06800000 \\
\hline $\mathrm{C}$ & 14.90100000 & 32.46700000 & 26.38200000 \\
\hline $\mathrm{C}$ & 15.12500000 & 31.79200000 & 25.08000000 \\
\hline $\mathrm{C}$ & 16.52300000 & 32.16000000 & 24.65500000 \\
\hline $\mathrm{N}$ & 10.84500000 & 23.40200000 & 20.04800000 \\
\hline $\mathrm{C}$ & 10.93400000 & 22.05000000 & 20.52400000 \\
\hline C & 9.58700000 & 21.52200000 & 20.96800000 \\
\hline 0 & 8.90700000 & 22.10500000 & 21.80400000 \\
\hline C & 11.88000000 & 21.91900000 & 21.72000000 \\
\hline $\mathrm{N}$ & 9.19800000 & 20.39700000 & 20.39600000 \\
\hline $\mathrm{C}$ & 7.93700000 & 19.78200000 & 20.82800000 \\
\hline C & 7.99900000 & 19.30100000 & 22.27200000 \\
\hline 0 & 7.04600000 & 19.36100000 & 23.02300000 \\
\hline $\mathrm{C}$ & 7.56400000 & 18.58900000 & 19.96900000 \\
\hline $\mathrm{C}$ & 7.30700000 & 18.86300000 & 18.51800000 \\
\hline 0 & 7.20500000 & 20.03400000 & 18.10400000 \\
\hline 0 & 7.20300000 & 17.87600000 & 17.76100000 \\
\hline $\mathrm{N}$ & 18.05000000 & 19.49400000 & 13.40100000 \\
\hline C & 18.68944002 & 19.83620428 & 12.12236296 \\
\hline $\mathrm{N}$ & 3.82100000 & 28.07100000 & 13.95400000 \\
\hline $\mathrm{C}$ & 4.07300000 & 28.25600000 & 12.54600000 \\
\hline $\mathrm{C}$ & 8.52800000 & 28.04900000 & 12.90000000 \\
\hline $\mathrm{C}$ & 9.21100000 & 29.15200000 & 13.67500000 \\
\hline 0 & 10.39700000 & 29.39300000 & 13.43800000 \\
\hline $\mathrm{N}$ & 17.62300000 & 31.59800000 & 28.75500000 \\
\hline $\mathrm{C}$ & 18.03900000 & 30.64400000 & 29.74800000 \\
\hline $\mathrm{N}$ & 19.93700000 & 31.34200000 & 15.21100000 \\
\hline C & 19.98300000 & 31.70100000 & 13.81600000 \\
\hline $\mathrm{N}$ & 7.61600000 & 22.65500000 & 24.22000000 \\
\hline C & 6.23500000 & 23.12700000 & 24.33400000 \\
\hline C & 5.39800000 & 22.31700000 & 25.31100000 \\
\hline 0 & 4.44200000 & 22.83900000 & 25.89900000 \\
\hline C & 5.55100000 & 23.11400000 & 22.94300000 \\
\hline $\mathrm{C}$ & 6.09100000 & 24.16500000 & 22.02400000 \\
\hline $\mathrm{C}$ & 5.33900000 & 24.37600000 & 20.71700000 \\
\hline $\mathrm{N}$ & 5.39700000 & 23.08400000 & 19.98000000 \\
\hline $\mathrm{C}$ & 4.44500000 & 22.17800000 & 19.97000000 \\
\hline $\mathrm{N}$ & 3.29400000 & 22.30000000 & 20.62500000 \\
\hline $\mathrm{N}$ & 4.61600000 & 21.04900000 & 19.26500000 \\
\hline $\mathrm{N}$ & 9.15900000 & 18.81300000 & 22.65900000 \\
\hline $\mathrm{C}$ & 9.43500000 & 18.05800000 & 23.85100000 \\
\hline $\mathrm{N}$ & 19.51200000 & 30.26200000 & 17.70400000 \\
\hline C & 20.23200000 & 31.51500000 & 17.59800000 \\
\hline $\mathrm{C}$ & 20.05200000 & 32.17500000 & 16.23600000 \\
\hline 0 & 20.02000000 & 33.39600000 & 16.11700000 \\
\hline $\mathrm{C}$ & 21.70600000 & 31.25600000 & 17.84000000 \\
\hline $\mathrm{C}$ & 22.12100000 & 30.72300000 & 19.19400000 \\
\hline 0 & 21.37800000 & 30.89500000 & 20.18000000 \\
\hline 0 & 23.23800000 & 30.12000000 & 19.20800000 \\
\hline $\mathrm{N}$ & 16.35900000 & 33.73100000 & 16.87100000 \\
\hline $\mathrm{C}$ & 16.33800000 & 34.93500000 & 17.68100000 \\
\hline C & 16.85500000 & 36.15300000 & 16.92600000 \\
\hline 0 & 16.44000000 & 37.28100000 & 17.21900000 \\
\hline $\mathrm{C}$ & 17.18000000 & 34.73200000 & 18.95100000 \\
\hline C & 16.55600000 & 33.79200000 & 19.96000000 \\
\hline $\mathrm{C}$ & 17.29100000 & 33.71200000 & 21.27500000 \\
\hline $\mathrm{N}$ & 18.69300000 & 33.31300000 & 21.08900000 \\
\hline $\mathrm{C}$ & 19.73600000 & 34.12100000 & 21.08000000 \\
\hline $\mathrm{N}$ & 19.66000000 & 35.44800000 & 21.24200000 \\
\hline
\end{tabular}

\begin{tabular}{|c|c|c|}
\hline 000 & 000 & 00 \\
\hline & 35.97100000 & \\
\hline 4000000 & 37.12000000 & 0000 \\
\hline & 21.04500000 & \\
\hline .89900000 & 20.25800000 & 44000000 \\
\hline 2.99700000 & 27.99100000 & 26.79400000 \\
\hline .90800000 & 27.95900000 & \\
\hline 3.28400000 & 28.93400000 & 24.63500000 \\
\hline .88000000 & 20.59300000 & 17.3650000 \\
\hline .90900000 & 19.12500000 & 17.7040000 \\
\hline 22.85900000 & 18.38500000 & 17.4780000 \\
\hline .58300000 & 32.28800000 & \\
\hline 5.65800000 & 33.67300000 & 15.74700000 \\
\hline .10500000 & 34.62800000 & 15.2300000 \\
\hline 6.72600000 & 18.17800000 & \\
\hline 17.44600000 & 18.34500000 & \\
\hline .43600000 & 17.45600000 & 12.6960000 \\
\hline 9.86600000 & 22.39700000 & 25.02000000 \\
\hline .40500000 & 22.68200000 & 25.2870000 \\
\hline 7.98600000 & 0000 & \\
\hline 18.02500000 & 20.9 & \\
\hline 11.16000000 & 30.14700000 & 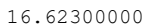 \\
\hline 11.95400000 & 29.24200000 & 17.4 \\
\hline 12.87700000 & 000 & 18. \\
\hline 13.96000000 & 30.4 & \\
\hline 12.74800000 & 28.36 & 16.4 \\
\hline 500000 & 27.2 & 17 \\
\hline 00000 & 27.3 & \\
\hline 13.9 & 00 & \\
\hline 12.43300000 & 30.1 & 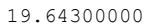 \\
\hline 13.23200000 & 30.8 & 0000 \\
\hline 14.5 & 30. & 20. \\
\hline 15.4 & 30.6 & \\
\hline 12 & & \\
\hline 11.21300000 & 32. & 2 \\
\hline & & \\
\hline & & \\
\hline 15.7 & 27. & \\
\hline & & \\
\hline 17.9 & 27 & \\
\hline 12.4 & 26.8 & \\
\hline 12.51700000 & 0000 & 23.2 \\
\hline 11.17400000 & 26.37 & 000 \\
\hline 10.6 & 25.2 & 22. \\
\hline 13 & 25. & 23. \\
\hline & 000 & . \\
\hline 13 & 25.7 & 10000 \\
\hline 14 & 24.2 & 00 \\
\hline 10.60 & 27.4 & 21. \\
\hline 0 & 27.2 & \\
\hline 41 & 26. & 100 \\
\hline 00 & 25. & 00 \\
\hline 72600000 & 28.5 & \\
\hline bo & 00 & \\
\hline & & \\
\hline 10.634 & 00 & 19. \\
\hline 10.886 & 000 & 0000 \\
\hline & 23.7 & \\
\hline 11.13 & 22. & 00 \\
\hline & 23. & 18. \\
\hline 00 & 0 & 000 \\
\hline 18.2 & 00 & 21. \\
\hline & 28 . & 21. \\
\hline & & \\
\hline 21.62000000 & 26.76200000 & 17.84800000 \\
\hline & & \\
\hline 20 & 00 & a \\
\hline & 25 . & \\
\hline & 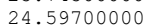 & 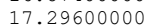 \\
\hline 18.90400000 & 23.48400000 & 16.76600000 \\
\hline & 00 & \\
\hline & 0 & \pm \\
\hline & 0000 & 17. \\
\hline & 0 & \\
\hline & 23.70200000 & 18.53600 \\
\hline & & \\
\hline & 2.8 & . \\
\hline 6900000 & 24.93600000 & 18.63500000 \\
\hline & & \\
\hline & 22.43200000 & 16 \\
\hline 14.1 & 22.15400000 & 15.30500000 \\
\hline & & \\
\hline 13.25100000 & 19.14500000 & 18.05300000 \\
\hline & & \\
\hline & 19.29900000 & 18.24800 \\
\hline 11 & 17.58600000 & 17.186000 \\
\hline & 17.05600000 & 16.7010000 \\
\hline
\end{tabular}




\section{Optimized Coordinates}

\section{Michaelis complex}

\begin{tabular}{|c|c|c|c|}
\hline C & -5.60018000 & 3.73995700 & 4.91818100 \\
\hline & -5.08588900 & 2.89227500 & 3.76917700 \\
\hline 0 & -5.31562500 & 3.20333300 & 2.59857900 \\
\hline $\mathrm{N}$ & -4.37439300 & 1.79677800 & 4.06609800 \\
\hline c & -3.55617800 & 1.14029000 & 3.06354300 \\
\hline c & -3.91040800 & -0.32904400 & 2.91138700 \\
\hline 0 & -3.44289700 & -1.16185600 & 3.68587200 \\
\hline C & -2.08062900 & 1.22964600 & 3.44931600 \\
\hline C & -1.22652700 & 0.41918800 & 2.45934300 \\
\hline 0 & -1.74504000 & 0.27655700 & 1.30764900 \\
\hline 0 & -0.12903600 & -0.00554800 & 2.85994400 \\
\hline $\mathrm{N}$ & -4.74477400 & -0.63590700 & 1.90141700 \\
\hline C & -5.15669700 & -2.00348800 & 1.69804700 \\
\hline C & -3.97197200 & -2.87894200 & 1.24395900 \\
\hline 0 & -4.06577000 & -4.11090500 & 1.32736700 \\
\hline c & -6.29854500 & -2.11702900 & 0.65934600 \\
\hline C & -7.53183800 & -1.33526100 & 1.14016300 \\
\hline 0 & -5.87985500 & -1.54362900 & -0.56131800 \\
\hline $\mathrm{N}$ & -2.88560700 & -2.29794700 & 0.77615900 \\
\hline C & -1.74123700 & -3.06849800 & 0.34253600 \\
\hline C & -0.73260900 & -3.36258600 & 1.44389500 \\
\hline 0 & 0.34031800 & -3.92047900 & 1.18916700 \\
\hline $\mathrm{N}$ & -1.10495800 & -3.02229500 & 2.68130200 \\
\hline c & -0.20279500 & -3.23131400 & 3.78016000 \\
\hline c & 8.50866100 & -3.27657400 & 0.18870200 \\
\hline C & 9.46433600 & -2.88629200 & -0.90914000 \\
\hline 0 & 10.63659300 & -3.23917400 & -0.95898800 \\
\hline $\mathrm{N}$ & 8.86727500 & -2.11534100 & -1.80199800 \\
\hline c & 9.71531400 & -1.47975400 & -2.75895700 \\
\hline C & -5.50290300 & -3.63058800 & -4.91957300 \\
\hline c & -4.97295300 & -2.83164500 & -3.74785700 \\
\hline 0 & -5.22164000 & -3.20237400 & -2.60215600 \\
\hline $\mathrm{N}$ & -4.25081800 & -1.74920200 & -4.01812600 \\
\hline c & -3.48170900 & -1.04845900 & -2.99992200 \\
\hline c & -3.84594700 & 0.42023500 & -2.86191000 \\
\hline 0 & -3.36014500 & 1.28430300 & -3.63412900 \\
\hline c & -2.01788800 & -1.17406800 & -3.42937800 \\
\hline c & -1.02745500 & -0.57163600 & -2.46328900 \\
\hline 0 & -1.36677700 & 0.05664000 & -1.47798600 \\
\hline o & 0.20392300 & -0.83024700 & -2.83618500 \\
\hline $\mathrm{N}$ & -4.70474600 & 0.75488100 & -1.88622700 \\
\hline c & -5.06092900 & 2.15742100 & -1.70247000 \\
\hline c & -3.90971400 & 3.03044200 & -1.17731900 \\
\hline 0 & -4.01179600 & 4.27418700 & -1.22965100 \\
\hline c & -6.25357900 & 2.28736200 & -0.72869900 \\
\hline c & -7.45170600 & 1.53785600 & -1.29039900 \\
\hline 0 & -5.88350900 & 1.70499400 & 0.52413300 \\
\hline $\mathrm{N}$ & -2.83795000 & 2.39722900 & -0.68306600 \\
\hline c & -1.64118900 & 3.07399400 & -0.24589200 \\
\hline$c$ & -0.58745600 & 3.24727300 & -1.33643900 \\
\hline 0 & 0.55624700 & 3.61120100 & -1.04978000 \\
\hline $\mathrm{N}$ & -0.99381300 & 3.05309900 & -2.59986700 \\
\hline c & -0.07825300 & 3.29202900 & -3.68873800 \\
\hline c & 8.18123400 & 2.91124100 & -0.22185000 \\
\hline c & 8.97370300 & 2.91259400 & 1.08625100 \\
\hline 0 & 9.92481400 & 3.66926600 & 1.25631600 \\
\hline $\mathrm{N}$ & 8.54390200 & 2.05369900 & 1.97094500 \\
\hline c & 9.12704700 & 2.01243800 & 3.30478500 \\
\hline c & 3.68547600 & -3.33459700 & 3.06797700 \\
\hline c & 3.91871200 & -2.50949600 & 1.81252700 \\
\hline 0 & 4.98421200 & -1.89457700 & 1.65323600 \\
\hline c & 3.12967600 & -2.00282800 & -0.45604100 \\
\hline c & 4.05221800 & 2.17617000 & -1.16262600 \\
\hline 0 & 5.19185800 & 1.77401500 & -1.40934500 \\
\hline $\mathrm{N}$ & 2.93400100 & -2.49718200 & 0.90060900 \\
\hline c & 2.89059700 & -0.49869800 & -0.63916900 \\
\hline C & 2.28486500 & -2.82602500 & -1.41675800 \\
\hline 0 & 2.37662900 & -0.02496500 & -1.66021000 \\
\hline 0 & 0.56719700 & -0.97995600 & 0.39981800 \\
\hline $\mathrm{N}$ & 3.43186600 & 0.31087300 & 0.28126600 \\
\hline c & 3.32610700 & 1.74940500 & 0.12214600 \\
\hline c & 3.99440700 & 2.42886900 & 1.31394000 \\
\hline $\mathrm{N}$ & 3.40810600 & 3.07004700 & -1.93204300 \\
\hline $\mathrm{c}$ & 4.01124800 & 3.55242400 & -3.16471900 \\
\hline 0 & 6.67536800 & 0.14467200 & 0.42631300 \\
\hline $\mathrm{H}$ & -1.91976800 & 0.82882300 & 4.45426700 \\
\hline $\mathrm{H}$ & -1.76035100 & 2.27824200 & 3.43776700 \\
\hline $\mathrm{H}$ & -3.72046800 & 1.65461300 & 2.11638300 \\
\hline $\mathrm{H}$ & -4.15206300 & 1.59776000 & 5.03274700 \\
\hline $\mathrm{H}$ & -6.51503700 & -3.18330000 & 0.53582000 \\
\hline $\mathrm{H}$ & -8.34881800 & -1.45711400 & 0.42310000 \\
\hline $\mathrm{H}$ & -7.86100800 & -1.69335400 & 2.12017800 \\
\hline $\mathrm{H}$ & -7.28996600 & -0.27028100 & 1.20874500 \\
\hline $\mathrm{H}$ & -5.58519400 & -2.23994700 & -1.18562300 \\
\hline $\mathrm{H}$ & -5.48142300 & -2.43755900 & 2.64925800 \\
\hline $\mathrm{H}$ & -5.06723200 & 0.09664800 & 1.26677600 \\
\hline $\mathrm{H}$ & -1.19819500 & -2.50586800 & -0.41928100 \\
\hline 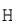 & -2.08573300 & -4.02242800 & -0.06276400 \\
\hline H & -2.73543900 & -1.28629600 & 0.84550900 \\
\hline & & & \\
\hline
\end{tabular}

\begin{tabular}{|c|c|c|}
\hline-1.76324900 & -2.22883900 & -3.56987800 \\
\hline-3.65116400 & -1.56409900 & -2.05030900 \\
\hline-4.07281600 & -1.49349400 & -4.98012400 \\
\hline-6.45796300 & 3.35624000 & -0.61012500 \\
\hline-8.31907900 & 1.66960000 & -0.63670100 \\
\hline-7.70320400 & 1.90821400 & -2.28890600 \\
\hline-7.23447000 & 0.46716000 & -1.35010800 \\
\hline-5.61622100 & 2.38811200 & 1.17614100 \\
\hline-5.32355700 & 2.58587000 & -2.67421800 \\
\hline-5.05801400 & 0.05010500 & -1.23228900 \\
\hline-1.19491500 & 2.49042400 & 0.56115400 \\
\hline-1.90436700 & 4.07224200 & 0.11530600 \\
\hline-2.80493100 & 1.38292600 & -0.62547800 \\
\hline 3.95777900 & 3.51500600 & 1.19961000 \\
\hline 5.04194500 & 2.11308700 & 1.37630100 \\
\hline 3.47977600 & 2.15714600 & 2.23983700 \\
\hline 2.26719500 & 2.02565800 & 0.05407300 \\
\hline 3.92682800 & -0.08689400 & 1.07226600 \\
\hline-5.48191000 & 3.25763000 & 5.89094000 \\
\hline-5.05038400 & 4.68475100 & 4.91662900 \\
\hline-6.65331300 & 3.96369100 & 4.74175100 \\
\hline-1.93789000 & -2.46264400 & 2.83522300 \\
\hline-0.75218600 & -3.10299100 & 4.71403600 \\
\hline 0.20812400 & -4.24280500 & 3.73160200 \\
\hline 0.61759200 & -2.50368000 & 3.75507900 \\
\hline 7.65830800 & -3.83055900 & -0.22045500 \\
\hline 9.01976900 & -3.89853400 & 0.92384400 \\
\hline 8.10939700 & -2.38553100 & 0.68447200 \\
\hline 8.02716700 & -1.62907200 & -1.51177500 \\
\hline 9.10988900 & -0.93449900 & -3.48570000 \\
\hline 10.42066300 & -0.78417800 & -2.28804600 \\
\hline 10.30008800 & -2.23834000 & -3.28219800 \\
\hline-6.58111500 & -3.74680200 & -4.79688600 \\
\hline-5.29138800 & -3.17813800 & -5.89130000 \\
\hline-5.05388500 & -4.62582800 & -4.88035100 \\
\hline-1.89567200 & 2.62400900 & -2.78644200 \\
\hline-0.61783700 & 3.18208000 & -4.63016300 \\
\hline 0.33045200 & 4.30452600 & -3.61970200 \\
\hline 0.75746000 & 2.58115900 & -3.66918500 \\
\hline 8.16974100 & 1.91276500 & -0.66931500 \\
\hline 7.13678300 & 3.19003300 & -0.05186300 \\
\hline 8.64350200 & 3.62088700 & -0.90752200 \\
\hline 7.84058100 & 1.37051600 & 1.68978200 \\
\hline 8.47860000 & 1.42710800 & 3.95822200 \\
\hline 10.12699900 & 1.56544900 & 3.29343400 \\
\hline 9.21763700 & 3.03086200 & 3.68903200 \\
\hline 2.73270300 & -3.86766500 & 3.03636800 \\
\hline 4.50900600 & -4.04687900 & 3.15671200 \\
\hline 3.71495100 & -2.67556200 & 3.93900500 \\
\hline 4.19809100 & -2.13656500 & -0.68082800 \\
\hline 2.02834400 & -2.93931000 & 1.09267600 \\
\hline 2.60094200 & -3.87157400 & -1.37774200 \\
\hline 1.23589200 & -2.76305500 & -1.11855900 \\
\hline 2.39496800 & -2.44884300 & -2.43502100 \\
\hline 0.91935100 & -0.48489500 & -2.21650600 \\
\hline-0.17717900 & -0.41779000 & 0.11401400 \\
\hline 2.42358100 & 3.26348500 & -1.73963900 \\
\hline 3.97962700 & 2.78818500 & -3.94904600 \\
\hline 3.46875600 & 4.43844200 & -3.49830700 \\
\hline 5.05606900 & 3.80891600 & -2.97816600 \\
\hline 6.09862300 & -0.55899900 & 0.77624200 \\
\hline 6.12681300 & 0.64369300 & -0.21687300 \\
\hline 0.54919600 & -0.78662200 & 1.36423200 \\
\hline
\end{tabular}
$0.54919600-0.78662200$
-128.6706758

1.36423200
Sum of electronic and zero-point Energies

Sum of electronic and thermal Free Energies: -3898.662512

\section{gem-Diol intermediate}

$\begin{array}{rrrr}\text { C } & 5.51796900 & -3.84816800 & 4.81748300 \\ \mathrm{C} & 5.01459400 & -2.96305000 & 3.69211400 \\ \mathrm{O} & 5.24919100 & -3.23953400 & 2.51383200 \\ \mathrm{~N} & 4.30723300 & -1.87325100 & 4.01849300 \\ \mathrm{C} & 3.49810900 & -1.18209900 & 3.03200200 \\ \mathrm{C} & 3.86094900 & 0.28928000 & 2.92667500 \\ \mathrm{O} & 3.39353600 & 1.10063600 & 3.72367200 \\ \mathrm{C} & 2.01652900 & -1.29898400 & 3.39960200 \\ \mathrm{C} & 1.17147000 & -0.66864300 & 2.31233400 \\ \mathrm{O} & 1.69266900 & -0.20430700 & 1.31092300 \\ \mathrm{O} & -0.11744000 & -0.70403000 & 2.53200200 \\ \mathrm{~N} & 4.70254000 & 0.62223200 & 1.93107200 \\ \mathrm{C} & 5.12283300 & 1.99310500 & 1.77173500 \\ \mathrm{C} & 3.94529400 & 2.88845200 & 1.33820500 \\ \mathrm{O} & 4.04514100 & 4.11676700 & 1.45967300 \\ \mathrm{C} & 6.27102300 & 2.13203000 & 0.74319200 \\ \mathrm{C} & 7.49746700 & 1.32924400 & 1.20658800 \\ \mathrm{O} & 5.85611700 & 1.59839100 & -0.49664200 \\ \mathrm{~N} & 2.85843900 & 2.32793700 & 0.84710600 \\ \mathrm{C} & 1.70103200 & 3.07003800 & 0.41824900 \\ \mathrm{C} & 0.70215200 & 3.33469300 & 1.53227500 \\ \mathrm{O} & -0.36246500 & 3.91169200 & 1.29788500 \\ \mathrm{~N} & 1.04829100 & 2.92167500 & 2.75814600 \\ \mathrm{C} & 0.16402700 & 3.18389100 & 3.86350400 \\ \mathrm{C} & -8.52762400 & 3.38670400 & 0.22859100\end{array}$




\begin{tabular}{|c|c|c|c|}
\hline $\mathrm{C}$ & -9.47824900 & 3.03479000 & -0.88654700 \\
\hline 0 & -10.64864300 & 3.39547600 & -0.93169700 \\
\hline $\mathrm{N}$ & -8.88070500 & 2.28803200 & -1.79892000 \\
\hline $\mathrm{C}$ & -9.72670000 & 1.68665000 & -2.77945500 \\
\hline C & 5.51448200 & 3.81927500 & -4.79116200 \\
\hline c & 4.97379800 & 2.98789100 & -3.64725600 \\
\hline 0 & 5.21805600 & 3.32214900 & -2.48944600 \\
\hline $\mathrm{N}$ & 4.24747300 & 1.91812800 & -3.95432700 \\
\hline $\mathrm{C}$ & 3.46904200 & 1.19095700 & -2.96221600 \\
\hline $\mathrm{C}$ & 3.82471900 & -0.28328000 & -2.86706100 \\
\hline 0 & 3.33859400 & -1.12075200 & -3.66787200 \\
\hline C & 2.00823300 & 1.32581200 & -3.39031300 \\
\hline C & 0.96658500 & 0.66334600 & -2.49084000 \\
\hline 0 & 1.34240400 & 0.17424200 & -1.38926600 \\
\hline 0 & -0.19963900 & 0.71729000 & -2.93705100 \\
\hline $\mathrm{N}$ & 4.67622100 & -0.65221000 & -1.89739000 \\
\hline C & 5.02396400 & -2.06161300 & -1.75461200 \\
\hline C & 3.86526800 & -2.94394200 & -1.26256700 \\
\hline 0 & 3.96105000 & -4.18605500 & -1.35227200 \\
\hline C & 6.21049300 & -2.22769400 & -0.77883900 \\
\hline C & 7.41566700 & -1.46796000 & -1.31091000 \\
\hline 0 & 5.83653200 & -1.68177700 & 0.48917200 \\
\hline $\mathrm{N}$ & 2.79412100 & -2.32026900 & -0.75503000 \\
\hline C & 1.61015900 & -3.04422200 & -0.36150300 \\
\hline C & 0.58243400 & -3.23304800 & -1.47008200 \\
\hline 0 & -0.52468400 & -3.70734800 & -1.20606000 \\
\hline $\mathrm{N}$ & 0.96953600 & -2.91690200 & -2.71075200 \\
\hline C & 0.04650200 & -3.10792300 & -3.80149200 \\
\hline C & -8.23101100 & -2.78952200 & -0.36999200 \\
\hline C & -9.02943200 & -2.82417800 & 0.93416700 \\
\hline 0 & -9.98587300 & -3.58105300 & 1.07557800 \\
\hline $\mathrm{N}$ & -8.60007600 & -1.99551000 & 1.84651200 \\
\hline C & -9.19062000 & -1.99198700 & 3.17801300 \\
\hline C & -3.71945400 & 3.32989000 & 3.13395000 \\
\hline C & -3.88521000 & 2.42756500 & 1.90926200 \\
\hline 0 & -4.95235400 & 1.83862900 & 1.71395600 \\
\hline C & -2.84728800 & 1.71381100 & -0.22872600 \\
\hline C & -3.88253000 & -2.11628800 & -1.18563200 \\
\hline 0 & -4.98141700 & -1.57815900 & -1.35498200 \\
\hline $\mathrm{N}$ & -2.82134700 & 2.33992600 & 1.09122200 \\
\hline C & -2.21471600 & 0.29665200 & -0.23489400 \\
\hline C & -2.15123700 & 2.63794600 & -1.22125800 \\
\hline 0 & -2.14710200 & -0.21825700 & -1.51045200 \\
\hline 0 & -0.89565900 & 0.41000400 & 0.32984000 \\
\hline $\mathrm{N}$ & -3.00199100 & -0.58249200 & 0.59959800 \\
\hline C & -3.11964500 & -1.96447200 & 0.14577400 \\
\hline C & -3.89247200 & -2.74572300 & 1.20861700 \\
\hline $\mathrm{N}$ & -3.33500800 & -2.95773200 & -2.08852900 \\
\hline C & -3.92581700 & -3.17241500 & -3.40324800 \\
\hline 0 & -6.57989900 & -0.20057600 & 0.53437100 \\
\hline $\mathrm{H}$ & 1.81362200 & -0.78257500 & 4.34362700 \\
\hline $\mathrm{H}$ & 1.72615800 & -2.34865400 & 3.51275200 \\
\hline $\mathrm{H}$ & 3.67526800 & -1.67095600 & 2.07158200 \\
\hline $\mathrm{H}$ & 4.10530700 & -1.68537700 & 4.99187600 \\
\hline $\mathrm{H}$ & 6.49309800 & 3.20068500 & 0.65619300 \\
\hline $\mathrm{H}$ & 8.31848500 & 1.47172500 & 0.49841500 \\
\hline $\mathrm{H}$ & 7.82324400 & 1.65283800 & 2.19976700 \\
\hline $\mathrm{H}$ & 7.25240600 & 0.26314400 & 1.23660300 \\
\hline $\mathrm{H}$ & 5.57616900 & 2.31156100 & -1.11271400 \\
\hline $\mathrm{H}$ & 5.44342700 & 2.39646300 & 2.73764100 \\
\hline $\mathrm{H}$ & 5.03530700 & -0.09389900 & 1.27978700 \\
\hline $\mathrm{H}$ & 1.18644200 & 2.47925000 & -0.34543600 \\
\hline $\mathrm{H}$ & 2.00689600 & 4.03320300 & 0.00287600 \\
\hline $\mathrm{H}$ & 2.78715300 & 1.31706300 & 0.76416200 \\
\hline $\mathrm{H}$ & 1.88809900 & 0.89656700 & -4.39047900 \\
\hline $\mathrm{H}$ & 1.75481200 & 2.38959800 & -3.45737100 \\
\hline $\mathrm{H}$ & 3.63723300 & 1.67387100 & -1.99645200 \\
\hline $\mathrm{H}$ & 4.04464200 & 1.71428600 & -4.92383600 \\
\hline $\mathrm{H}$ & 6.40940300 & -3.30031200 & -0.68903400 \\
\hline $\mathrm{H}$ & 8.27876200 & -1.62228500 & -0.65629600 \\
\hline $\mathrm{H}$ & 7.67116600 & -1.81137700 & -2.31777900 \\
\hline $\mathrm{H}$ & 7.20298300 & -0.39527800 & -1.34314100 \\
\hline $\mathrm{H}$ & 5.56783800 & -2.38848400 & 1.11080900 \\
\hline $\mathrm{H}$ & 5.29042500 & -2.46181800 & -2.73729000 \\
\hline $\mathrm{H}$ & 5.02948400 & 0.03128600 & -1.22385600 \\
\hline $\mathrm{H}$ & 1.10718500 & -2.51144200 & 0.44744500 \\
\hline $\mathrm{H}$ & 1.90260800 & -4.03839400 & -0.01449800 \\
\hline $\mathrm{H}$ & 2.69295200 & -1.30601900 & -0.81099900 \\
\hline $\mathrm{H}$ & -4.00132700 & -3.79488400 & 0.91760900 \\
\hline $\mathrm{H}$ & -4.89294400 & -2.31216200 & 1.32664800 \\
\hline $\mathrm{H}$ & -3.36860800 & -2.68676700 & 2.16554900 \\
\hline $\mathrm{H}$ & -2.11289500 & -2.37818700 & 0.03005700 \\
\hline $\mathrm{H}$ & -3.92504100 & -0.18854700 & 0.77030100 \\
\hline $\mathrm{H}$ & 5.41320500 & -3.38921900 & 5.80303600 \\
\hline $\mathrm{H}$ & 4.94947000 & -4.78156300 & 4.79406900 \\
\hline $\mathrm{H}$ & 6.56538800 & -4.08725800 & 4.62923700 \\
\hline $\mathrm{H}$ & 1.92409500 & 2.43332200 & 2.91567400 \\
\hline $\mathrm{H}$ & 0.68708200 & 2.95545800 & 4.79321500 \\
\hline $\mathrm{H}$ & -0.13999600 & 4.23421900 & 3.86197600 \\
\hline $\mathrm{H}$ & -0.73800900 & 2.56316300 & 3.80236900 \\
\hline $\mathrm{H}$ & -7.70192600 & 3.99632300 & -0.15134300 \\
\hline $\mathrm{H}$ & -9.05453300 & 3.94610100 & 1.00194100 \\
\hline $\mathrm{H}$ & -8.09128300 & 2.48426800 & 0.66907400 \\
\hline $\mathrm{H}$ & -8.02640700 & 1.81138900 & -1.53971100 \\
\hline $\mathrm{H}$ & -9.12031900 & 1.16029800 & -3.51913800 \\
\hline
\end{tabular}

\begin{tabular}{|c|c|c|c|}
\hline $\mathrm{H}$ & -10.43704900 & 0.98069500 & -2.33240900 \\
\hline $\mathrm{H}$ & -10.30548900 & 2.46324000 & -3.28271100 \\
\hline $\mathrm{H}$ & 6.59423900 & 3.91740900 & -4.66488400 \\
\hline $\mathrm{H}$ & 5.29795800 & 3.40116500 & -5.77695200 \\
\hline $\mathrm{H}$ & 5.07860600 & 4.81858300 & -4.72000200 \\
\hline $\mathrm{H}$ & 1.83213200 & -2.40392200 & -2.87095600 \\
\hline $\mathrm{H}$ & 0.58012000 & -2.96242100 & -4.74156100 \\
\hline $\mathrm{H}$ & -0.36378200 & -4.12070900 & -3.76566200 \\
\hline $\mathrm{H}$ & -0.78054300 & -2.38939900 & -3.74608400 \\
\hline $\mathrm{H}$ & -8.19526400 & -1.77437600 & -0.77691900 \\
\hline $\mathrm{H}$ & -7.19318000 & -3.09582300 & -0.20445800 \\
\hline $\mathrm{H}$ & -8.70424800 & -3.46334500 & -1.08367100 \\
\hline $\mathrm{H}$ & -7.84582200 & -1.34862100 & 1.60898100 \\
\hline $\mathrm{H}$ & -8.57747700 & -1.37306100 & 3.83434200 \\
\hline $\mathrm{H}$ & -10.21198600 & -1.59756700 & 3.15848900 \\
\hline $\mathrm{H}$ & -9.23227100 & -3.01310400 & 3.56603200 \\
\hline $\mathrm{H}$ & -2.80068100 & 3.92002600 & 3.09223800 \\
\hline $\mathrm{H}$ & -4.58636200 & 3.99167100 & 3.18384400 \\
\hline $\mathrm{H}$ & -3.71858400 & 2.70793000 & 4.03313000 \\
\hline $\mathrm{H}$ & -3.89876000 & 1.58379600 & -0.50877300 \\
\hline $\mathrm{H}$ & -1.98245200 & 2.87893000 & 1.30766400 \\
\hline $\mathrm{H}$ & -2.64098800 & 3.61553400 & -1.22061400 \\
\hline $\mathrm{H}$ & -1.10599100 & 2.78459500 & -0.93297700 \\
\hline $\mathrm{H}$ & -2.18010100 & 2.21923100 & -2.22810400 \\
\hline $\mathrm{H}$ & -1.39212500 & 0.17794600 & -2.04193200 \\
\hline $\mathrm{H}$ & -0.17569100 & 0.19090900 & -0.31589400 \\
\hline $\mathrm{H}$ & -2.36592100 & -3.23512000 & -1.93323900 \\
\hline $\mathrm{H}$ & -3.66560100 & -2.36481800 & -4.09755600 \\
\hline $\mathrm{H}$ & -3.56981900 & -4.12405300 & -3.80321000 \\
\hline $\mathrm{H}$ & -5.01169000 & -3.20067700 & -3.29752300 \\
\hline $\mathrm{H}$ & -6.02802000 & 0.50105800 & 0.92781300 \\
\hline $\mathrm{H}$ & -6.01249600 & -0.64906500 & -0.12936600 \\
\hline $\mathrm{H}$ & -0.58191800 & -0.28249400 & 1.72401600 \\
\hline
\end{tabular}

Sum of electronic and zero-point Energies: -3898.577839

Sum of electronic and thermal Free Energies: -3898.654335

\section{$\mathrm{N}$-protonated gem-diol}

\begin{tabular}{|c|c|c|c|}
\hline C & 5.48981500 & -3.72294300 & 4.88743500 \\
\hline $\mathrm{C}$ & 4.98110700 & -2.85510400 & 3.75105900 \\
\hline 0 & 5.21747700 & -3.14490000 & 2.57633300 \\
\hline $\mathrm{N}$ & 4.26704800 & -1.76570100 & 4.06373000 \\
\hline C & 3.45379900 & -1.09183100 & 3.06873000 \\
\hline $\mathrm{C}$ & 3.80759200 & 0.38023100 & 2.94487700 \\
\hline 0 & 3.33518400 & 1.19863800 & 3.73168000 \\
\hline $\mathrm{C}$ & 1.95736000 & -1.24534900 & 3.37717500 \\
\hline C & 1.05024600 & -0.80484700 & 2.20144300 \\
\hline 0 & 1.59351800 & -0.14225500 & 1.28666000 \\
\hline 0 & -0.14713900 & -1.18786100 & 2.25665700 \\
\hline $\mathrm{N}$ & 4.64721300 & 0.70583000 & 1.94521200 \\
\hline $\mathrm{C}$ & 5.05906200 & 2.07714000 & 1.76872500 \\
\hline C & 3.87616200 & 2.95975100 & 1.32387400 \\
\hline 0 & 3.96842400 & 4.19008100 & 1.43003300 \\
\hline $\mathrm{C}$ & 6.20649400 & 2.21020100 & 0.73847300 \\
\hline $\mathrm{C}$ & 7.43779300 & 1.42081600 & 1.21188300 \\
\hline 0 & 5.79493600 & 1.65852800 & -0.49458300 \\
\hline $\mathrm{N}$ & 2.79278700 & 2.38647400 & 0.83989900 \\
\hline $\mathrm{C}$ & 1.62967200 & 3.11992100 & 0.40578600 \\
\hline $\mathrm{C}$ & 0.60341300 & 3.35104000 & 1.50451100 \\
\hline 0 & -0.49048200 & 3.86955700 & 1.25514100 \\
\hline $\mathrm{N}$ & 0.97788500 & 3.00730600 & 2.74191900 \\
\hline $\mathrm{C}$ & 0.09289100 & 3.26366800 & 3.84547500 \\
\hline $\mathrm{C}$ & -8.59921800 & 3.36713000 & 0.20796700 \\
\hline $\mathrm{C}$ & -9.54811600 & 2.99599700 & -0.90221300 \\
\hline 0 & -10.72050900 & 3.34874700 & -0.95197700 \\
\hline $\mathrm{N}$ & -8.94572100 & 2.24146700 & -1.80531300 \\
\hline $\mathrm{C}$ & -9.78796200 & 1.62263400 & -2.77820800 \\
\hline $\mathrm{C}$ & 5.43998000 & 3.82325500 & -4.81660500 \\
\hline $\mathrm{C}$ & 4.90438800 & 3.00292800 & -3.66234400 \\
\hline 0 & 5.14644300 & 3.35323100 & -2.50882800 \\
\hline $\mathrm{N}$ & 4.18463900 & 1.92486500 & -3.95608100 \\
\hline $\mathrm{C}$ & 3.41056800 & 1.20575800 & -2.95462100 \\
\hline $\mathrm{C}$ & 3.77534400 & -0.26514100 & -2.84135400 \\
\hline 0 & 3.29436300 & -1.11555600 & -3.63144400 \\
\hline $\mathrm{C}$ & 1.96037800 & 1.32717500 & -3.41606800 \\
\hline $\mathrm{C}$ & 0.93334000 & 0.58788300 & -2.57756300 \\
\hline 0 & 1.29351200 & 0.05160600 & -1.50728100 \\
\hline 0 & -0.23056100 & 0.62311400 & -3.06629600 \\
\hline $\mathrm{N}$ & 4.62895700 & -0.61666500 & -1.86699700 \\
\hline $\mathrm{C}$ & 4.98523400 & -2.02198700 & -1.70646400 \\
\hline $\mathrm{C}$ & 3.83212800 & -2.90518800 & -1.20327400 \\
\hline 0 & 3.93539600 & -4.14770700 & -1.27766000 \\
\hline $\mathrm{C}$ & 6.17282200 & -2.16855400 & -0.72882700 \\
\hline $\mathrm{C}$ & 7.37331800 & -1.40817700 & -1.27033200 \\
\hline 0 & 5.79539000 & -1.60910800 & 0.53230600 \\
\hline $\mathrm{N}$ & 2.75697900 & -2.28178300 & -0.70391600 \\
\hline $\mathrm{C}$ & 1.59519700 & -3.04266400 & -0.30913200 \\
\hline $\mathrm{C}$ & 0.64389800 & -3.37981000 & -1.44835100 \\
\hline 0 & -0.39049100 & -4.02474400 & -1.22537700 \\
\hline $\mathrm{N}$ & 0.95281500 & -2.92344800 & -2.66440500 \\
\hline $\mathrm{C}$ & 0.01453000 & -3.12438500 & -3.74006100 \\
\hline $\mathrm{C}$ & -8.26411500 & -2.81228400 & -0.31225800 \\
\hline $\mathrm{C}$ & -9.06368000 & -2.83742800 & 0.99143400 \\
\hline
\end{tabular}




\begin{tabular}{|c|c|c|c|}
\hline 0 & -10.01506900 & -3.59785900 & 1.14261300 \\
\hline $\mathrm{N}$ & -8.63927500 & -1.99450600 & 1.89374000 \\
\hline $\mathrm{C}$ & -9.22983700 & -1.97759200 & 3.22489000 \\
\hline C & -3.79140200 & 3.37671700 & 3.11411200 \\
\hline C & -3.96863600 & 2.36087300 & 1.99021200 \\
\hline 0 & -5.02626000 & 1.73428800 & 1.88171000 \\
\hline C & -3.03177500 & 1.59786300 & -0.16648900 \\
\hline $\mathrm{C}$ & -3.45234200 & -2.19265300 & -1.12009400 \\
\hline 0 & -4.53555200 & -1.63191700 & -0.96012600 \\
\hline $\mathrm{N}$ & -2.92234300 & 2.21156800 & 1.15236900 \\
\hline $\mathrm{C}$ & -1.99803500 & 0.47347000 & -0.37532100 \\
\hline C & -2.80509900 & 2.66974000 & -1.23236700 \\
\hline 0 & -2.06946700 & -0.04427900 & -1.61454900 \\
\hline 0 & -0.78640300 & 0.95223900 & 0.03974500 \\
\hline $\mathrm{N}$ & -2.37811600 & -0.73102500 & 0.59564600 \\
\hline $\mathrm{C}$ & -2.34278800 & -2.07161800 & -0.05735100 \\
\hline C & -2.47956400 & -3.15749700 & 1.00440900 \\
\hline $\mathrm{N}$ & -3.15102900 & -3.02321300 & -2.14450000 \\
\hline $\mathrm{C}$ & -4.01151100 & -3.29096800 & -3.30582800 \\
\hline 0 & -6.62114900 & -0.16446400 & 0.49200300 \\
\hline $\mathrm{H}$ & 1.70078800 & -0.64074500 & 4.25524200 \\
\hline $\mathrm{H}$ & 1.73217700 & -2.29065500 & 3.61056100 \\
\hline $\mathrm{H}$ & 3.66185300 & -1.58234700 & 2.11516400 \\
\hline $\mathrm{H}$ & 4.04254500 & -1.58469400 & 5.03356900 \\
\hline $\mathrm{H}$ & 6.42272000 & 3.27889700 & 0.63669800 \\
\hline $\mathrm{H}$ & 8.25820900 & 1.55758700 & 0.50159900 \\
\hline $\mathrm{H}$ & 7.76138700 & 1.76012200 & 2.20053000 \\
\hline $\mathrm{H}$ & 7.19722800 & 0.35428200 & 1.25771800 \\
\hline $\mathrm{H}$ & 5.50175000 & 2.36278400 & -1.11286800 \\
\hline $\mathrm{H}$ & 5.37722900 & 2.49457000 & 2.72953400 \\
\hline $\mathrm{H}$ & 4.97485000 & -0.01459700 & 1.29822600 \\
\hline $\mathrm{H}$ & 1.12921700 & 2.55591000 & -0.38613500 \\
\hline $\mathrm{H}$ & 1.93808000 & 4.09808900 & 0.02852800 \\
\hline $\mathrm{H}$ & 2.67836600 & 1.37046400 & 0.86511400 \\
\hline $\mathrm{H}$ & 1.87614000 & 0.94055800 & -4.43729300 \\
\hline $\mathrm{H}$ & 1.67784900 & 2.38512000 & -3.44271300 \\
\hline $\mathrm{H}$ & 3.56445100 & 1.70399200 & -1.99430700 \\
\hline $\mathrm{H}$ & 3.99076600 & 1.70103700 & -4.92273500 \\
\hline $\mathrm{H}$ & 6.37824400 & -3.23898400 & -0.62683000 \\
\hline $\mathrm{H}$ & 8.23724400 & -1.54887600 & -0.61368500 \\
\hline $\mathrm{H}$ & 7.63107400 & -1.76208300 & -2.27314000 \\
\hline $\mathrm{H}$ & 7.15366500 & -0.33728400 & -1.31505300 \\
\hline $\mathrm{H}$ & 5.52479400 & -2.30636400 & 1.16573700 \\
\hline $\mathrm{H}$ & 5.25386500 & -2.43223500 & -2.68443900 \\
\hline $\mathrm{H}$ & 4.97215100 & 0.07577000 & -1.19658800 \\
\hline $\mathrm{H}$ & 1.01569600 & -2.47100900 & 0.42140100 \\
\hline $\mathrm{H}$ & 1.91107300 & -3.98500900 & 0.14477200 \\
\hline $\mathrm{H}$ & 2.65371900 & -1.26801100 & -0.71679400 \\
\hline $\mathrm{H}$ & -2.31335000 & -4.12935900 & 0.53534000 \\
\hline $\mathrm{H}$ & -3.47825100 & -3.13694100 & 1.45477000 \\
\hline $\mathrm{H}$ & -1.72032400 & -3.00843700 & 1.77765900 \\
\hline $\mathrm{H}$ & -1.36730900 & -2.14098600 & -0.53158300 \\
\hline $\mathrm{H}$ & -3.30294500 & -0.56919400 & 1.01043700 \\
\hline $\mathrm{H}$ & 5.37345400 & -3.25517100 & 5.86756300 \\
\hline $\mathrm{H}$ & 4.93418200 & -4.66418500 & 4.87051700 \\
\hline $\mathrm{H}$ & 6.54166000 & -3.94985900 & 4.70798100 \\
\hline $\mathrm{H}$ & 1.84222400 & 2.49684800 & 2.89959900 \\
\hline $\mathrm{H}$ & 0.63032100 & 3.07933700 & 4.77672600 \\
\hline $\mathrm{H}$ & -0.24828200 & 4.30241600 & 3.81882400 \\
\hline $\mathrm{H}$ & -0.78865200 & 2.61117200 & 3.81027100 \\
\hline $\mathrm{H}$ & -7.78486200 & 3.98777200 & -0.17882100 \\
\hline $\mathrm{H}$ & -9.13140700 & 3.92340200 & 0.97991600 \\
\hline $\mathrm{H}$ & -8.14836800 & 2.47386400 & 0.65224500 \\
\hline $\mathrm{H}$ & -8.08984400 & 1.77213400 & -1.53772600 \\
\hline $\mathrm{H}$ & -9.17835700 & 1.10123500 & -3.51892900 \\
\hline $\mathrm{H}$ & -10.48668700 & 0.90931300 & -2.32434700 \\
\hline $\mathrm{H}$ & -10.37962000 & 2.38863500 & -3.28236100 \\
\hline $\mathrm{H}$ & 6.51930200 & 3.92843800 & -4.69229200 \\
\hline $\mathrm{H}$ & 5.22512400 & 3.39241600 & -5.79737800 \\
\hline $\mathrm{H}$ & 4.99907400 & 4.82110400 & -4.75703600 \\
\hline $\mathrm{H}$ & 1.75134900 & -2.30730000 & -2.80047800 \\
\hline $\mathrm{H}$ & 0.52094600 & -2.93634500 & -4.68808600 \\
\hline $\mathrm{H}$ & -0.35225600 & -4.15337400 & -3.72001900 \\
\hline $\mathrm{H}$ & -0.83413600 & -2.43324800 & -3.65609600 \\
\hline $\mathrm{H}$ & -8.20910900 & -1.79545500 & -0.71265700 \\
\hline $\mathrm{H}$ & -7.23521200 & -3.14521900 & -0.14154300 \\
\hline $\mathrm{H}$ & -8.74893400 & -3.47437500 & -1.02902800 \\
\hline $\mathrm{H}$ & -7.89863800 & -1.33804300 & 1.64382400 \\
\hline $\mathrm{H}$ & -8.61870500 & -1.35015800 & 3.87487700 \\
\hline $\mathrm{H}$ & -10.25210800 & -1.58625900 & 3.20083800 \\
\hline $\mathrm{H}$ & -9.26813600 & -2.99472500 & 3.62292600 \\
\hline $\mathrm{H}$ & -2.84430700 & 3.91594900 & 3.03766400 \\
\hline $\mathrm{H}$ & -4.62453200 & 4.08090200 & 3.05806100 \\
\hline $\mathrm{H}$ & -3.85280700 & 2.86197300 & 4.07626400 \\
\hline $\mathrm{H}$ & -4.03084300 & 1.16031200 & -0.26237700 \\
\hline $\mathrm{H}$ & -2.08423600 & 2.78472300 & 1.29201300 \\
\hline $\mathrm{H}$ & -3.59107200 & 3.42680400 & -1.16561500 \\
\hline $\mathrm{H}$ & -1.83751000 & 3.15098000 & -1.05818400 \\
\hline $\mathrm{H}$ & -2.80998200 & 2.22302600 & -2.22790200 \\
\hline $\mathrm{H}$ & -1.23055800 & 0.22287800 & -2.21506000 \\
\hline $\mathrm{H}$ & -0.02427600 & 0.36248400 & -0.19013200 \\
\hline $\mathrm{H}$ & -2.22127300 & -3.44385700 & -2.11535800 \\
\hline $\mathrm{H}$ & -3.52908900 & -2.93597600 & -4.22054800 \\
\hline $\mathrm{H}$ & -4.21758600 & -4.36100400 & -3.38907300 \\
\hline $\mathrm{H}$ & -4.94579300 & -2.74941800 & -3.15316100 \\
\hline
\end{tabular}

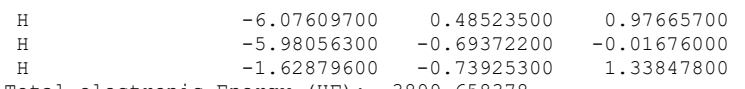

Total electronic Energy (HF) : -3899.658378

Sum of electronic and zero-point Energies: -3898.571685

Sum of electronic and thermal Free Energies: -3898.649645

\section{Product complex}

\begin{tabular}{|c|c|c|c|}
\hline C & -5.44210100 & -3.82634600 & -4.80907100 \\
\hline $\mathrm{C}$ & -4.95051900 & -2.93553100 & -3.68298800 \\
\hline 0 & -5.18839200 & -3.21174000 & -2.50529600 \\
\hline $\mathrm{N}$ & -4.24987100 & -1.84103700 & -4.00805000 \\
\hline C & -3.45046900 & -1.14205300 & -3.01914800 \\
\hline $\mathrm{C}$ & -3.82480800 & 0.32668100 & -2.91804200 \\
\hline 0 & -3.35987300 & 1.14014900 & -3.71434200 \\
\hline C & -1.97506900 & -1.29277400 & -3.39489400 \\
\hline C & -1.00131000 & -0.89682000 & -2.28093700 \\
\hline 0 & -1.48060300 & -0.12839700 & -1.36810600 \\
\hline 0 & 0.14453100 & -1.34445900 & -2.32453700 \\
\hline $\mathrm{N}$ & -4.67339200 & 0.65502800 & -1.92685800 \\
\hline C & -5.10465400 & 2.02297900 & -1.77189400 \\
\hline C & -3.93587200 & 2.92789100 & -1.33454500 \\
\hline 0 & -4.04438700 & 4.15520900 & -1.45861000 \\
\hline C & -6.25855500 & 2.15505500 & -0.74878100 \\
\hline $\mathrm{C}$ & -7.47681600 & 1.34228000 & -1.21633100 \\
\hline 0 & -5.84531000 & 1.62669100 & 0.49386900 \\
\hline $\mathrm{N}$ & -2.84715400 & 2.37638700 & -0.83741400 \\
\hline $\mathrm{C}$ & -1.71591900 & 3.16776600 & -0.41762100 \\
\hline C & -0.70271500 & 3.43835500 & -1.52013500 \\
\hline 0 & 0.35132600 & 4.03025500 & -1.27394400 \\
\hline $\mathrm{N}$ & -1.04457900 & 3.02188400 & -2.74419700 \\
\hline C & -0.14541300 & 3.24736000 & -3.84323100 \\
\hline C & 8.52742600 & 3.52121900 & -0.16877500 \\
\hline $\mathrm{C}$ & 9.47614100 & 3.17900500 & 0.95079200 \\
\hline 0 & 10.64336900 & 3.54833200 & 1.00073500 \\
\hline $\mathrm{N}$ & 8.87981100 & 2.42925400 & 1.86192700 \\
\hline C & 9.72583500 & 1.83589300 & 2.84733000 \\
\hline $\mathrm{C}$ & -5.53990400 & 3.85749900 & 4.78597700 \\
\hline $\mathrm{C}$ & -4.98788200 & 3.02815700 & 3.64599700 \\
\hline 0 & -5.22926600 & 3.35864400 & 2.48650800 \\
\hline $\mathrm{N}$ & -4.25488100 & 1.96440400 & 3.95831300 \\
\hline C & -3.46649700 & 1.24154300 & 2.97088900 \\
\hline C & -3.81073000 & -0.23557600 & 2.87686500 \\
\hline 0 & -3.32194800 & -1.06800000 & 3.68128600 \\
\hline C & -2.00389600 & 1.35352800 & 3.40499700 \\
\hline C & -1.11390700 & 0.58038600 & 2.46940100 \\
\hline 0 & -1.48307100 & 0.27684400 & 1.34945700 \\
\hline 0 & 0.06740500 & 0.30668400 & 2.97449400 \\
\hline $\mathrm{N}$ & -4.65495400 & -0.61257600 & 1.90391700 \\
\hline C & -4.99144400 & -2.02478200 & 1.76204700 \\
\hline C & -3.82404100 & -2.89927100 & 1.27662900 \\
\hline 0 & -3.91080500 & -4.14187900 & 1.36834900 \\
\hline $\mathrm{C}$ & -6.17227600 & -2.20146500 & 0.78119500 \\
\hline $\mathrm{C}$ & -7.38551700 & -1.44987500 & 1.30643000 \\
\hline 0 & -5.79662700 & -1.65494200 & -0.48607500 \\
\hline $\mathrm{N}$ & -2.75514500 & -2.26840800 & 0.77320500 \\
\hline $\mathrm{C}$ & -1.55820500 & -2.97900300 & 0.37655200 \\
\hline $\mathrm{C}$ & -0.56379800 & -3.21664300 & 1.50493900 \\
\hline 0 & 0.50796400 & -3.78270400 & 1.28157000 \\
\hline $\mathrm{N}$ & -0.91117100 & -2.79031600 & 2.72876300 \\
\hline $\mathrm{C}$ & -0.01568000 & -3.03014400 & 3.83333700 \\
\hline C & 8.27383100 & -2.65411200 & 0.43840300 \\
\hline C & 9.07969800 & -2.68698100 & -0.86122800 \\
\hline 0 & 10.04203600 & -3.43642200 & -0.99723600 \\
\hline $\mathrm{N}$ & 8.64816400 & -1.86280600 & -1.77738000 \\
\hline $\mathrm{C}$ & 9.24460100 & -1.85689500 & -3.10599600 \\
\hline C & 3.73351700 & 3.42377300 & -3.09620700 \\
\hline C & 3.91878300 & 2.51590000 & -1.89017000 \\
\hline 0 & 4.99653900 & 1.95353300 & -1.69340500 \\
\hline $\mathrm{C}$ & 2.81273800 & 1.55197100 & 0.08344500 \\
\hline $\mathrm{C}$ & 3.81557000 & -2.29411100 & 1.13568900 \\
\hline 0 & 4.90227300 & -1.71868000 & 1.23810000 \\
\hline $\mathrm{N}$ & 2.83635800 & 2.38405300 & -1.10184700 \\
\hline $\mathrm{C}$ & 1.57826900 & 0.66171100 & 0.12430700 \\
\hline C & 2.81700700 & 2.41026400 & 1.35523500 \\
\hline 0 & 1.51552500 & -0.26308100 & 0.93606200 \\
\hline 0 & 0.62115300 & 1.04350300 & -0.66273700 \\
\hline $\mathrm{N}$ & 3.41458400 & -1.32415800 & -1.07401000 \\
\hline C & 3.06803400 & -2.43096500 & -0.19196800 \\
\hline $\mathrm{C}$ & 3.35992700 & -3.82083700 & -0.77762600 \\
\hline $\mathrm{N}$ & 3.25289100 & -2.94241700 & 2.18094100 \\
\hline $\mathrm{C}$ & 3.90185000 & -3.00685400 & 3.48153400 \\
\hline 0 & 6.41361500 & -0.29453000 & -0.67732100 \\
\hline $\mathrm{H}$ & -1.76552500 & -0.66466600 & -4.26924700 \\
\hline $\mathrm{H}$ & -1.76837600 & -2.33181600 & -3.66575200 \\
\hline $\mathrm{H}$ & -3.63671100 & -1.63045300 & -2.05989900 \\
\hline $\mathrm{H}$ & -4.01690900 & -1.67318200 & -4.97829600 \\
\hline $\mathrm{H}$ & -6.48982100 & 3.22184400 & -0.66260400 \\
\hline H & -8.30273400 & 1.47838400 & -0.51235600 \\
\hline $\mathrm{H}$ & -7.79977400 & 1.66312400 & -2.21116700 \\
\hline H & -7.22272500 & 0.27835900 & -1.24593000 \\
\hline $\mathrm{H}$ & -5.56773900 & 2.34588300 & 1.10026300 \\
\hline $\mathrm{H}$ & -5.42459100 & 2.42234600 & -2.73977400 \\
\hline
\end{tabular}




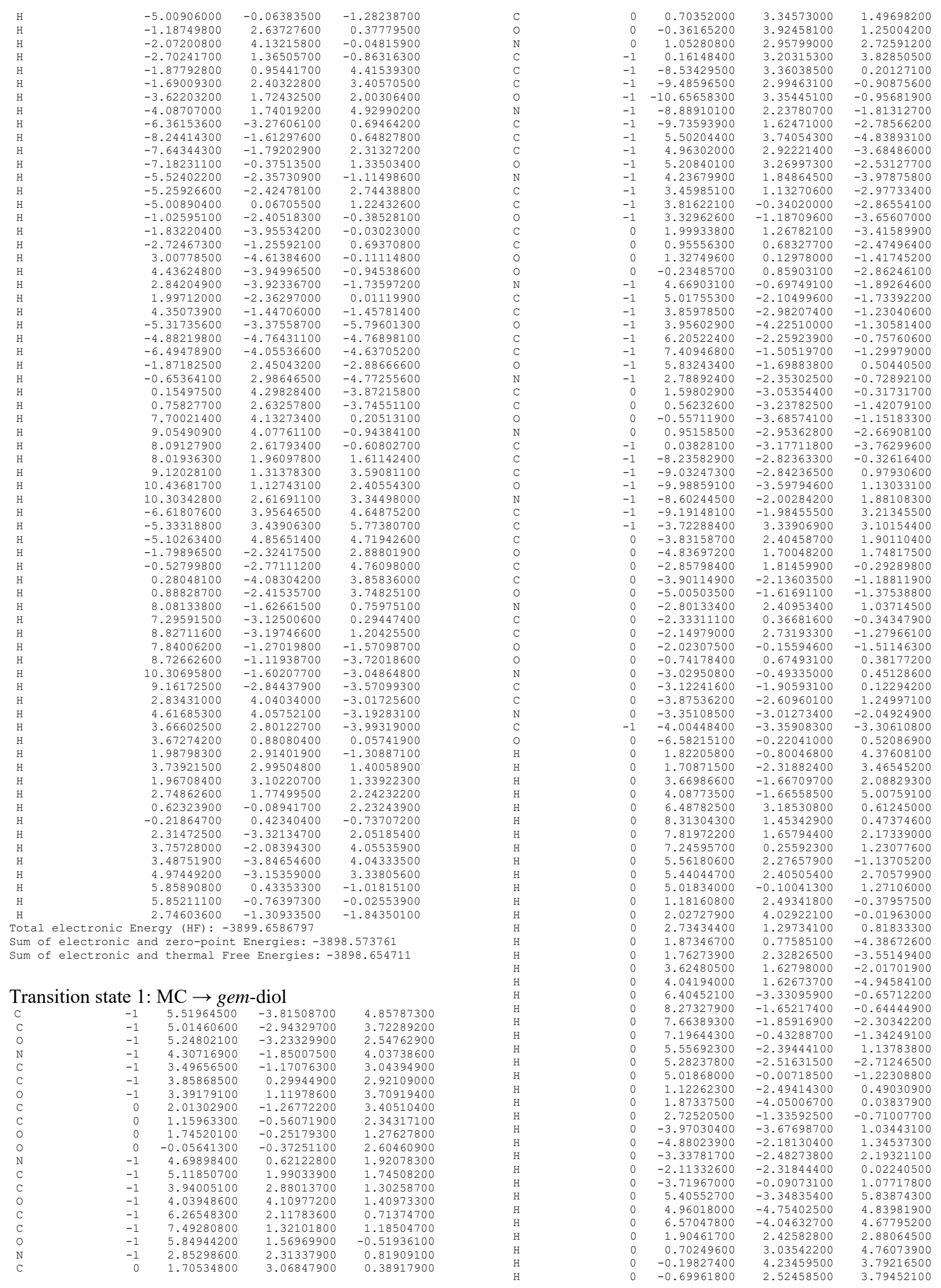




\begin{tabular}{|c|c|c|c|}
\hline $\mathrm{H}$ & 0 & -7.71176400 & 3.96921700 \\
\hline $\mathrm{H}$ & 0 & -9.06139300 & 3.92570900 \\
\hline $\mathrm{H}$ & 0 & -8.09665400 & 2.46216500 \\
\hline $\mathrm{H}$ & 0 & -8.03738000 & 1.76058200 \\
\hline $\mathrm{H}$ & 0 & -9.13013300 & 1.09190900 \\
\hline $\mathrm{H}$ & 0 & -10.44429700 & 0.92232800 \\
\hline $\mathrm{H}$ & 0 & -10.31679800 & 2.39518800 \\
\hline $\mathrm{H}$ & 0 & 6.58168200 & 3.84162700 \\
\hline $\mathrm{H}$ & 0 & 5.28574900 & 3.31076900 \\
\hline $\mathrm{H}$ & 0 & 5.06483500 & 4.74000800 \\
\hline $\mathrm{H}$ & 0 & 1.82785200 & -2.46594200 \\
\hline $\mathrm{H}$ & 0 & 0.57467200 & -3.03183500 \\
\hline $\mathrm{H}$ & 0 & -0.35200600 & -4.19794900 \\
\hline $\mathrm{H}$ & 0 & -0.80614400 & -2.47789300 \\
\hline $\mathrm{H}$ & 0 & -8.19340200 & -1.81262900 \\
\hline $\mathrm{H}$ & 0 & -7.20035600 & -3.13659100 \\
\hline $\mathrm{H}$ & 0 & -8.71405000 & -3.50095300 \\
\hline $\mathrm{H}$ & 0 & -7.85273700 & -1.35576100 \\
\hline $\mathrm{H}$ & 0 & -8.57289100 & -1.36594500 \\
\hline $\mathrm{H}$ & 0 & -10.20976100 & -1.58249000 \\
\hline $\mathrm{H}$ & 0 & -9.24014100 & -3.00267300 \\
\hline $\mathrm{H}$ & 0 & -2.80525200 & 3.93128700 \\
\hline $\mathrm{H}$ & 0 & -4.59199700 & 4.00081600 \\
\hline $\mathrm{H}$ & 0 & -3.76580700 & 2.74505800 \\
\hline $\mathrm{H}$ & 0 & -3.91873900 & 1.72228200 \\
\hline $\mathrm{H}$ & 0 & -1.96652800 & 2.96862500 \\
\hline $\mathrm{H}$ & 0 & -2.63271800 & 3.71255200 \\
\hline $\mathrm{H}$ & 0 & -1.10337900 & 2.86167000 \\
\hline $\mathrm{H}$ & 0 & -2.19226300 & 2.32489800 \\
\hline $\mathrm{H}$ & 0 & -1.24806400 & 0.33102100 \\
\hline $\mathrm{H}$ & 0 & 0.00242500 & 0.31131800 \\
\hline $\mathrm{H}$ & 0 & -2.38262700 & -3.29533200 \\
\hline $\mathrm{H}$ & 0 & -3.85799300 & -2.57953400 \\
\hline $\mathrm{H}$ & 0 & -3.58995000 & -4.30042200 \\
\hline $\mathrm{H}$ & 0 & -5.07585600 & -3.47076600 \\
\hline $\mathrm{H}$ & 0 & -6.01146500 & 0.45807000 \\
\hline $\mathrm{H}$ & 0 & -6.01727700 & -0.67489100 \\
\hline $\mathrm{H}$ & 0 & -0.59162000 & 0.25966800 \\
\hline Tot & En & ergy (HF): -38 & 9.651183 \\
\hline Sum & $c$ a & nd zero-point & hergies: -389 \\
\hline Sum & $c$ a & nd thermal Free & Energies: -3 \\
\hline Ima & ncy & $:-209.66$ & \\
\hline & & & \\
\hline & & & \\
\hline $\operatorname{Tr}$ & e 2 & gem-diol - & $N$-protonat \\
\hline $\mathrm{C}$ & -1 & 5.47386400 & -3.35417900 \\
\hline $\mathrm{C}$ & -1 & 4.96554300 & -2.56600000 \\
\hline 0 & -1 & 5.20409000 & -2.93420100 \\
\hline $\mathrm{N}$ & -1 & 4.24934300 & -1.45906500 \\
\hline $\mathrm{C}$ & -1 & 3.43636200 & -0.85549000 \\
\hline $\mathrm{C}$ & -1 & 3.78827900 & 0.60545200 \\
\hline 0 & -1 & 3.31330900 & 1.47441400 \\
\hline $\mathrm{C}$ & 0 & 1.93098100 & -0.99352800 \\
\hline $\mathrm{C}$ & 0 & 1.04475800 & -0.61093600 \\
\hline 0 & 0 & 1.55239700 & -0.02184600 \\
\hline 0 & 0 & -0.18898800 & -0.98626600 \\
\hline $\mathrm{N}$ & -1 & 4.62877600 & 0.86410500 \\
\hline $\mathrm{C}$ & -1 & 5.03877900 & 2.22103200 \\
\hline $\mathrm{C}$ & -1 & 3.85512100 & 3.06958400 \\
\hline 0 & -1 & 3.94537200 & 4.30441600 \\
\hline $\mathrm{C}$ & -1 & 6.18754600 & 2.28601500 \\
\hline $\mathrm{C}$ & -1 & 7.41936600 & 1.53249300 \\
\hline 0 & -1 & 5.77872200 & 1.65153700 \\
\hline $\mathrm{N}$ & -1 & 2.77345900 & 2.46306000 \\
\hline $\mathrm{C}$ & 0 & 1.60612800 & 3.16154400 \\
\hline $\mathrm{C}$ & 0 & 0.56803900 & 3.43834400 \\
\hline 0 & 0 & -0.54004400 & 3.90247200 \\
\hline $\mathrm{N}$ & 0 & 0.95315900 & 3.19643600 \\
\hline $\mathrm{C}$ & -1 & 0.06764200 & 3.53702500 \\
\hline $\mathrm{C}$ & -1 & -8.61887500 & 3.37963200 \\
\hline $\mathrm{C}$ & -1 & -9.56588700 & 2.93338600 \\
\hline 0 & -1 & -10.73867700 & 3.27947200 \\
\hline $\mathrm{N}$ & -1 & -8.96081700 & 2.12010300 \\
\hline $\mathrm{C}$ & $\begin{array}{l}1 \\
-1\end{array}$ & -9.80067000 & 1.43544600 \\
\hline $\mathrm{C}$ & -1 & 5.42691800 & 3.51845400 \\
\hline $\mathrm{C}$ & -1 & 4.89082600 & 2.777720600 \\
\hline o & -1 & 5.13062100 & 3.20507600 \\
\hline $\mathrm{N}$ & -1 & 4.17315900 & 1.68067800 \\
\hline $\mathrm{C}$ & -1 & 3.39876500 & 1.02931500 \\
\hline $\mathrm{C}$ & -1 & 3.76559100 & -0.42975200 \\
\hline 0 & -1 & 3.28718600 & -1.33245000 \\
\hline $\mathrm{C}$ & 0 & 1.94004600 & 1.08693200 \\
\hline $\mathrm{C}$ & 0 & 1.00265500 & 0.26859700 \\
\hline 0 & 0 & 1.37708700 & 0.04028600 \\
\hline 0 & 0 & -0.07675500 & -0.07531700 \\
\hline $\mathrm{N}$ & -1 & 4.61836800 & -0.71316800 \\
\hline $\mathrm{C}$ & -1 & 4.97666600 & -2.10384800 \\
\hline $\mathrm{C}$ & $\begin{array}{l}1 \\
-1\end{array}$ & 3.82400400 & -2.95290400 \\
\hline 0 & -1 & 3.92944100 & -4.19742300 \\
\hline $\mathrm{C}$ & -1 & 6.16288500 & -2.18198600 \\
\hline $\mathrm{C}$ & -1 & 7.36304200 & -1.45798300 \\
\hline 0 & -1 & 5.78272100 & -1.53914800 \\
\hline $\mathrm{N}$ & -1 & 2.74727500 & -2.29891000 \\
\hline $\mathrm{C}$ & 0 & 1.60041600 & -3.05972600 \\
\hline & & & \\
\hline
\end{tabular}




$\begin{array}{rrrrr}\mathrm{H} & 0 & -1.24059400 & -0.14474100 & -2.14940900 \\ \mathrm{H} & 0 & 0.01573900 & 0.47272900 & -0.35416200 \\ \mathrm{H} & 0 & -2.16662500 & -3.56253000 & -1.95929000 \\ \mathrm{H} & 0 & -3.45701100 & -3.09564500 & -4.06216100 \\ \mathrm{H} & 0 & -4.21317600 & -4.45640900 & -3.18245600 \\ \mathrm{H} & 0 & -4.86322400 & -2.80568100 & -3.00336000 \\ \mathrm{H} & 0 & -6.06943500 & 0.55731700 & 0.95014600 \\ \mathrm{H} & 0 & -5.88555800 & -0.74536200 & 0.13501900 \\ \mathrm{H} & 0 & -1.02389300 & -0.77027700 & 1.48311700\end{array}$

Total electronic Energy (HF) : -3899.651533

Sum of electronic and zero-point Energies: -3898.568356

Sum of electronic and thermal Free Energies: -3898.643524

Imaginary frequency: -1085.01

Transition state 3: $\mathrm{N}$-protonated $\mathrm{gem}$-diol $\rightarrow$ product complex

\begin{tabular}{|c|c|c|c|c|}
\hline & & & & \\
\hline C & -1 & 5.49149800 & -3.82487600 & 4.80898600 \\
\hline C & -1 & 4.98608500 & -2.93352600 & 3.68947000 \\
\hline 0 & -1 & 5.22335800 & -3.20039900 & 2.50950200 \\
\hline $\mathrm{N}$ & -1 & 4.27398000 & -1.84899800 & 4.02285100 \\
\hline $\mathrm{C}$ & -1 & 3.46343100 & -1.15369700 & 3.04044700 \\
\hline C & -1 & 3.82058100 & 0.31973500 & 2.94638500 \\
\hline 0 & -1 & 3.34883400 & 1.12336100 & 3.74866400 \\
\hline $\mathrm{C}$ & 0 & 1.97239300 & -1.31153100 & 3.35596000 \\
\hline C & 0 & 1.05791600 & -0.90188800 & 2.17882800 \\
\hline 0 & 0 & 1.57796500 & -0.16957900 & 1.28539400 \\
\hline 0 & 0 & -0.10052400 & -1.35813200 & 2.18549100 \\
\hline $\mathrm{N}$ & -1 & 4.66210700 & 0.66335300 & 1.95438000 \\
\hline $\mathrm{C}$ & -1 & 5.07713700 & 2.03700500 & 1.80575100 \\
\hline C & -1 & 3.89670800 & 2.93084300 & 1.37702400 \\
\hline 0 & -1 & 3.99143200 & 4.15861400 & 1.50780700 \\
\hline C & -1 & 6.22616800 & 2.18805900 & 0.77976700 \\
\hline C & -1 & 7.45516900 & 1.38675300 & 1.23891400 \\
\hline 0 & -1 & 5.81503600 & 1.66190000 & -0.46452200 \\
\hline $\mathrm{N}$ & -1 & 2.81271400 & 2.36964500 & 0.88045100 \\
\hline C & 0 & 1.65404500 & 3.11818200 & 0.46190300 \\
\hline C & 0 & 0.62886900 & 3.33968900 & 1.56362500 \\
\hline 0 & 0 & -0.45801800 & 3.87242400 & 1.31776100 \\
\hline $\mathrm{N}$ & 0 & 0.99970800 & 2.96650700 & 2.79274100 \\
\hline $\mathrm{C}$ & -1 & 0.11082800 & 3.19271100 & 3.89949700 \\
\hline C & -1 & -8.57628300 & 3.38725100 & 0.25389200 \\
\hline $\mathrm{C}$ & -1 & -9.52464000 & 3.04035800 & -0.86455700 \\
\hline 0 & -1 & -10.69618500 & 3.39650400 & -0.90875500 \\
\hline $\mathrm{N}$ & -1 & -8.92264400 & 2.30263200 & -1.78176500 \\
\hline $\mathrm{C}$ & -1 & -9.76496400 & 1.70507800 & -2.76781600 \\
\hline C & -1 & 5.47026700 & 3.91292600 & -4.74307200 \\
\hline $\mathrm{C}$ & -1 & 4.93139700 & 3.07100200 & -3.60604400 \\
\hline 0 & -1 & 5.17276900 & 3.39770900 & -2.44547500 \\
\hline $\mathrm{N}$ & -1 & 4.20973500 & 2.00065300 & -3.92193600 \\
\hline C & -1 & 3.43279300 & 1.26314600 & -2.93624700 \\
\hline $\mathrm{C}$ & -1 & 3.79438700 & -0.21032600 & -2.85152100 \\
\hline 0 & -1 & 3.31253600 & -1.04384000 & -3.65903000 \\
\hline C & 0 & 1.98413000 & 1.38153700 & -3.40315400 \\
\hline C & 0 & 1.01514800 & 0.61036400 & -2.54889800 \\
\hline 0 & 0 & 1.33338100 & 0.16308300 & -1.45649700 \\
\hline 0 & 0 & -0.16353500 & 0.52345500 & -3.09686700 \\
\hline $\mathrm{N}$ & -1 & 4.64594900 & -0.58301600 & -1.88335600 \\
\hline $\mathrm{C}$ & -1 & 4.99908400 & -1.99201900 & -1.75050000 \\
\hline C & -1 & 3.84345300 & -2.88257300 & -1.26615400 \\
\hline 0 & -1 & 3.94409100 & -4.12357300 & -1.36531800 \\
\hline C & -1 & 6.18502800 & -2.16058100 & -0.77435600 \\
\hline C & -1 & 7.38785900 & -1.39216400 & -1.29917000 \\
\hline 0 & -1 & 5.80718600 & -1.62548000 & 0.49714300 \\
\hline $\mathrm{N}$ & -1 & 2.76887600 & -2.26689400 & -0.75608900 \\
\hline $\mathrm{C}$ & 0 & 1.59815100 & -3.02469900 & -0.37119000 \\
\hline C & 0 & 0.62576800 & -3.29818700 & -1.50918500 \\
\hline 0 & 0 & -0.42516900 & -3.91192100 & -1.29634400 \\
\hline $\mathrm{N}$ & 0 & 0.95149200 & -2.83879100 & -2.72367100 \\
\hline $\mathrm{C}$ & -1 & 0.02861000 & -3.04300100 & -3.81169400 \\
\hline $\mathrm{C}$ & -1 & -8.25374600 & -2.78130100 & -0.38867900 \\
\hline $\mathrm{C}$ & -1 & -9.05508300 & -2.83063800 & 0.91327000 \\
\hline 0 & -1 & -10.00828200 & -3.59183500 & 1.04807400 \\
\hline $\mathrm{N}$ & -1 & -8.62985200 & -2.00675500 & 1.83266800 \\
\hline $\mathrm{C}$ & -1 & -9.22227000 & -2.01503500 & 3.16315300 \\
\hline $\mathrm{C}$ & -1 & -3.77229300 & 3.32857900 & 3.16566000 \\
\hline C & 0 & -3.99500700 & 2.41056700 & 1.97002700 \\
\hline 0 & 0 & -5.08122200 & 1.84594700 & 1.81431800 \\
\hline $\mathrm{C}$ & 0 & -3.01654800 & 1.64519100 & -0.16741700 \\
\hline C & 0 & -3.57342400 & -2.16674800 & -1.14930000 \\
\hline 0 & 0 & -4.65955900 & -1.58661500 & -1.10498500 \\
\hline $\mathrm{N}$ & 0 & -2.94841900 & 2.28091700 & 1.13624100 \\
\hline $\mathrm{C}$ & 0 & -1.93435100 & 0.58074000 & -0.35973300 \\
\hline $\mathrm{C}$ & 0 & -2.80882700 & 2.70615100 & -1.25572300 \\
\hline 0 & 0 & -1.89582400 & -0.03437800 & -1.45428100 \\
\hline 0 & 0 & -0.84528100 & 0.88497600 & 0.35099300 \\
\hline $\mathrm{N}$ & 0 & -2.73505300 & -0.91266600 & 0.80430200 \\
\hline $\mathrm{C}$ & 0 & -2.59783300 & -2.15233800 & 0.03856100 \\
\hline C & 0 & -2.79640800 & -3.40184600 & 0.89973900 \\
\hline $\mathrm{N}$ & 0 & -3.18163300 & -2.94370200 & -2.18890700 \\
\hline C & -1 & -3.96159800 & -3.15184400 & -3.41023800 \\
\hline 0 & 0 & -6.55403600 & -0.22013100 & 0.56276700 \\
\hline $\mathrm{H}$ & 0 & 1.71862200 & -0.69363400 & 4.22561300 \\
\hline $\mathrm{H}$ & 0 & 1.75441800 & -2.35415700 & 3.60511700 \\
\hline
\end{tabular}

\begin{tabular}{|c|c|c|c|c|}
\hline $\mathrm{H}$ & 0 & 3.66996600 & -1.62779300 & 2.07830100 \\
\hline $\mathrm{H}$ & 0 & 4.04354000 & -1.69051800 & 4.99528600 \\
\hline H & 0 & 6.44493200 & 3.25794900 & 0.69872700 \\
\hline $\mathrm{H}$ & 0 & 8.27705400 & 1.53570400 & 0.53277400 \\
\hline H & 0 & 7.77790100 & 1.70603800 & 2.23438100 \\
\hline $\mathrm{H}$ & 0 & 7.21250600 & 0.32004000 & 1.26390400 \\
\hline $\mathrm{H}$ & 0 & 5.52568300 & 2.38067100 & -1.06619800 \\
\hline H & 0 & 5.39529700 & 2.43483700 & 2.77484200 \\
\hline H & 0 & 4.99448200 & -0.04625200 & 1.29846400 \\
\hline $\mathrm{H}$ & 0 & 1.14805900 & 2.56801400 & -0.33615300 \\
\hline $\mathrm{H}$ & 0 & 1.96851000 & 4.09930800 & 0.09780700 \\
\hline $\mathrm{H}$ & 0 & 2.68311600 & 1.35412300 & 0.90906200 \\
\hline $\mathrm{H}$ & 0 & 1.89554200 & 1.00554000 & -4.42717000 \\
\hline $\mathrm{H}$ & 0 & 1.67996600 & 2.43395900 & -3.40535400 \\
\hline $\mathrm{H}$ & 0 & 3.57905200 & 1.74442800 & -1.96596700 \\
\hline H & 0 & 4.03563200 & 1.78294200 & -4.89377800 \\
\hline $\mathrm{H}$ & 0 & 6.38720500 & -3.23339600 & -0.69440200 \\
\hline $\mathrm{H}$ & 0 & 8.25053900 & -1.54854600 & -0.64459700 \\
\hline $\mathrm{H}$ & 0 & 7.64635000 & -1.72590300 & -2.30870400 \\
\hline $\mathrm{H}$ & 0 & 7.17172500 & -0.31980500 & -1.32149000 \\
\hline $\mathrm{H}$ & 0 & 5.53959000 & -2.33405700 & 1.12072400 \\
\hline $\mathrm{H}$ & 0 & 5.26826400 & -2.38311500 & -2.73610000 \\
\hline H & 0 & 4.99350500 & 0.09614300 & -1.20041000 \\
\hline $\mathrm{H}$ & 0 & 1.03648400 & -2.48035200 & 0.39160400 \\
\hline $\mathrm{H}$ & 0 & 1.90772200 & -3.99103700 & 0.03413600 \\
\hline $\mathrm{H}$ & 0 & 2.70622400 & -1.25542800 & -0.67097100 \\
\hline $\mathrm{H}$ & 0 & -2.58727000 & -4.30127300 & 0.31482300 \\
\hline H & 0 & -3.82109100 & -3.45014600 & 1.28550000 \\
\hline $\mathrm{H}$ & 0 & -2.09411600 & -3.36567300 & 1.73771900 \\
\hline $\mathrm{H}$ & 0 & -1.57900200 & -2.16303700 & -0.34994300 \\
\hline $\mathrm{H}$ & 0 & -3.69536200 & -0.77236500 & 1.12225300 \\
\hline $\mathrm{H}$ & 0 & 5.37063400 & -3.37820700 & 5.79831300 \\
\hline $\mathrm{H}$ & 0 & 4.93721300 & -4.76623600 & 4.76971700 \\
\hline $\mathrm{H}$ & 0 & 6.54438300 & -4.04659600 & 4.62891600 \\
\hline $\mathrm{H}$ & 0 & 1.85505600 & 2.43804400 & 2.93832000 \\
\hline $\mathrm{H}$ & 0 & 0.64359700 & 2.97879000 & 4.82705200 \\
\hline $\mathrm{H}$ & 0 & -0.22646300 & 4.23296700 & 3.90253700 \\
\hline $\mathrm{H}$ & 0 & -0.77234600 & 2.54456900 & 3.83922600 \\
\hline $\mathrm{H}$ & 0 & -7.75205300 & 4.00202300 & -0.12107900 \\
\hline $\mathrm{H}$ & 0 & -9.10527900 & 3.94036200 & 1.03027300 \\
\hline $\mathrm{H}$ & 0 & -8.13702700 & 2.48429100 & 0.69029200 \\
\hline $\mathrm{H}$ & 0 & -8.06588400 & 1.82940300 & -1.52507400 \\
\hline $\mathrm{H}$ & 0 & -9.15555200 & 1.19793000 & -3.51852400 \\
\hline $\mathrm{H}$ & 0 & -10.46465800 & 0.98331600 & -2.32917300 \\
\hline $\mathrm{H}$ & 0 & -10.35529400 & 2.48221200 & -3.25630200 \\
\hline $\mathrm{H}$ & 0 & 6.54876500 & 4.01762700 & -4.61231800 \\
\hline $\mathrm{H}$ & 0 & 5.25981500 & 3.50061300 & -5.73271100 \\
\hline $\mathrm{H}$ & 0 & 5.02725000 & 4.90863100 & -4.66609800 \\
\hline $\mathrm{H}$ & 0 & 1.79591200 & -2.29215300 & -2.86721400 \\
\hline $\mathrm{H}$ & 0 & 0.53052200 & -2.79647900 & -4.74862400 \\
\hline $\mathrm{H}$ & 0 & -0.29678500 & -4.08652500 & -3.83397100 \\
\hline $\mathrm{H}$ & 0 & -0.85345500 & -2.39923300 & -3.70614400 \\
\hline $\mathrm{H}$ & 0 & -8.14407400 & -1.75140200 & -0.74095800 \\
\hline $\mathrm{H}$ & 0 & -7.24317600 & -3.17272100 & -0.23281100 \\
\hline $\mathrm{H}$ & 0 & -8.76733400 & -3.38599500 & -1.13565000 \\
\hline $\mathrm{H}$ & 0 & -7.86664500 & -1.36575800 & 1.60716700 \\
\hline $\mathrm{H}$ & 0 & -8.65427800 & -1.34155600 & 3.80596100 \\
\hline $\mathrm{H}$ & 0 & -10.26673700 & -1.68958600 & 3.12870400 \\
\hline $\mathrm{H}$ & 0 & -9.19949900 & -3.02663400 & 3.57814600 \\
\hline $\mathrm{H}$ & 0 & -2.83269100 & 3.88158800 & 3.09317800 \\
\hline $\mathrm{H}$ & 0 & -4.61203200 & 4.02465700 & 3.21725800 \\
\hline $\mathrm{H}$ & 0 & -3.77810300 & 2.72942400 & 4.08029300 \\
\hline $\begin{array}{l}\mathrm{H} \\
\mathrm{H}\end{array}$ & 0 & -3.99055000 & 1.16492000 & -0.27809200 \\
\hline $\mathrm{H}$ & 0 & -2.08720900 & 2.79833300 & 1.32860400 \\
\hline $\mathrm{H}$ & 0 & -3.61631800 & 3.44200600 & -1.21700300 \\
\hline $\mathrm{H}$ & 0 & -1.85901500 & 3.22361000 & -1.08019700 \\
\hline $\mathrm{H}$ & 0 & -2.78789800 & 2.23594000 & -2.24120700 \\
\hline $\mathrm{H}$ & 0 & -0.85078900 & 0.18199100 & -2.38610400 \\
\hline $\mathrm{H}$ & 0 & -0.08124500 & 0.26280800 & 0.25239800 \\
\hline $\mathrm{H}$ & 0 & -2.25491600 & -3.36396600 & -2.11210800 \\
\hline $\mathrm{H}$ & 0 & -3.45077700 & -2.71704100 & -4.27505600 \\
\hline $\mathrm{H}$ & 0 & -4.12315500 & -4.21936400 & -3.58350200 \\
\hline $\mathrm{H}$ & 0 & -4.92247600 & -2.65463200 & -3.27243700 \\
\hline $\mathrm{H}$ & 0 & -6.03287800 & 0.48945700 & 0.98576400 \\
\hline $\mathrm{H}$ & 0 & -5.92141000 & -0.70239100 & -0.00352900 \\
\hline $\mathrm{H}$ & 0 & -2.08229900 & -0.94144600 & 1.59460400 \\
\hline
\end{tabular}

Total electronic Energy (HF) : -3899.651643

Sum of electronic and zero-point Energies: -3898.56634

Sum of electronic and thermal Free Energies: -3898.643802

Imaginary frequency: -147.83

\section{Product complex + constrained water}

$\begin{array}{llrl}\mathrm{C} & -5.46808100 & -3.39027500 & -5.16924500 \\ \mathrm{C} & -4.97413500 & -2.62363100 & -3.95617400 \\ \mathrm{O} & -5.21452900 & -3.01994000 & -2.81381500 \\ \mathrm{~N} & -4.26858400 & -1.50422200 & -4.16500200 \\ \mathrm{C} & -3.46745400 & -0.91538700 & -3.10811300 \\ \mathrm{C} & -3.83582900 & 0.53641300 & -2.85491200 \\ \mathrm{O} & -3.36659900 & 1.42638600 & -3.56184800 \\ \mathrm{C} & -1.99316700 & -1.03267000 & -3.50058100 \\ \mathrm{C} & -1.01019100 & -0.74902500 & -2.36174000 \\ \mathrm{O} & -1.48161400 & -0.06962200 & -1.37451600 \\ \mathrm{O} & 0.13440000 & -1.18878100 & -2.45637000\end{array}$




\begin{tabular}{|c|c|c|c|}
\hline $\mathrm{N}$ & -4.68420900 & 0.76337500 & -1.83555100 \\
\hline C & -5.10998400 & 2.10957100 & -1.53932500 \\
\hline $\mathrm{C}$ & -3.93797300 & 2.95910000 & -1.00926500 \\
\hline 0 & -4.04126800 & 4.19308800 & -1.00497200 \\
\hline C & -6.26452700 & 2.13930300 & -0.50886700 \\
\hline C & -7.48559400 & 1.38479400 & -1.05939100 \\
\hline 0 & -5.85492900 & 1.48269000 & 0.67232300 \\
\hline $\mathrm{N}$ & -2.85214400 & 2.35417000 & -0.57147300 \\
\hline C & -1.72114600 & 3.09152300 & -0.07176300 \\
\hline C & -0.71202400 & 3.45596100 & -1.14324000 \\
\hline 0 & 0.34571800 & 4.03264200 & -0.83656400 \\
\hline $\mathrm{N}$ & -1.03298300 & 3.15384800 & -2.39650900 \\
\hline C & -0.14329500 & 3.52194200 & -3.46827300 \\
\hline C & 8.52629200 & 3.37498200 & 0.22107600 \\
\hline C & 9.47227000 & 2.91405100 & 1.29962400 \\
\hline 0 & 10.64095800 & 3.27122100 & 1.38860500 \\
\hline $\mathrm{N}$ & 8.87177700 & 2.07604900 & 2.12730900 \\
\hline $\mathrm{C}$ & 9.71418300 & 1.37974000 & 3.04619800 \\
\hline C & -5.54531900 & 3.25320300 & 5.17358900 \\
\hline C & -4.99539900 & 2.54472000 & 3.95386200 \\
\hline 0 & -5.23405600 & 2.99514700 & 2.83490700 \\
\hline $\mathrm{N}$ & -4.26717200 & 1.45113600 & 4.15426500 \\
\hline C & -3.48063200 & 0.83167100 & 3.09752600 \\
\hline C & -3.83086500 & -0.62616000 & 2.84996900 \\
\hline 0 & -3.34647400 & -1.53987300 & 3.56370800 \\
\hline C & -2.01633300 & 0.89134900 & 3.53576400 \\
\hline C & -1.13515300 & 0.22260500 & 2.51484400 \\
\hline 0 & -1.51235200 & 0.04724800 & 1.36993900 \\
\hline 0 & 0.04732700 & -0.11196100 & 2.97781200 \\
\hline $\mathrm{N}$ & -4.67550200 & -0.89623500 & 1.84243800 \\
\hline C & -5.01766600 & -2.28455800 & 1.55406000 \\
\hline C & -3.85332500 & -3.10869600 & 0.98108800 \\
\hline 0 & -3.94533800 & -4.35372100 & 0.94287200 \\
\hline C & -6.19807100 & -2.35315600 & 0.55927700 \\
\hline C & -7.40880500 & -1.65519900 & 1.15901900 \\
\hline 0 & -5.81867900 & -1.67926200 & -0.64392800 \\
\hline $\mathrm{N}$ & -2.78123900 & -2.43338500 & 0.54686800 \\
\hline C & -1.58555300 & -3.10076800 & 0.07863300 \\
\hline C & -0.59436800 & -3.46185500 & 1.17705200 \\
\hline 0 & 0.47467900 & -4.00682400 & 0.89619100 \\
\hline $\mathrm{N}$ & -0.94137600 & -3.16356500 & 2.43835800 \\
\hline C & -0.04853500 & -3.52117900 & 3.51306900 \\
\hline C & 8.24643100 & -2.82887300 & 0.18182000 \\
\hline C & 9.05367600 & -2.72967200 & -1.11357900 \\
\hline 0 & 10.01306300 & -3.46495100 & -1.32617000 \\
\hline $\mathrm{N}$ & 8.62663000 & -1.81277400 & -1.93925900 \\
\hline $\mathrm{C}$ & 9.22464100 & -1.67109700 & -3.25960000 \\
\hline C & 3.73545100 & 3.60316400 & -2.70410900 \\
\hline C & 3.92262600 & 2.57036800 & -1.60409100 \\
\hline 0 & 5.00113600 & 1.99364000 & -1.46541100 \\
\hline C & 2.81403400 & 1.36906500 & 0.23195200 \\
\hline C & 3.79604900 & -2.54717400 & 0.90174900 \\
\hline 0 & 4.88323700 & -1.98656700 & 1.06369500 \\
\hline $\mathrm{N}$ & 2.83846300 & 2.34548800 & -0.83824500 \\
\hline C & 1.55359000 & 0.51645600 & 0.18349500 \\
\hline C & 2.88180900 & 2.04781900 & 1.60561100 \\
\hline 0 & 1.47568900 & -0.49829700 & 0.87841700 \\
\hline 0 & 0.59914300 & 1.01120400 & -0.54041600 \\
\hline $\mathrm{N}$ & 3.41929300 & -1.37497300 & -1.20978800 \\
\hline $\mathrm{C}$ & 3.05478800 & -2.55448400 & -0.43591500 \\
\hline C & 3.33520400 & -3.88776900 & -1.14555100 \\
\hline $\mathrm{N}$ & 3.22608400 & -3.28951900 & 1.87768500 \\
\hline $\mathrm{C}$ & 3.86946700 & -3.47799200 & 3.16847800 \\
\hline 0 & 6.40057500 & -0.36340900 & -0.68244700 \\
\hline $\mathrm{H}$ & -1.78535400 & -0.32334000 & -4.31099600 \\
\hline $\mathrm{H}$ & -1.79092200 & -2.04076900 & -3.87260500 \\
\hline $\mathrm{H}$ & -3.65626100 & -1.50068400 & -2.20520400 \\
\hline $\mathrm{H}$ & -4.03553600 & -1.23579400 & -5.11235800 \\
\hline $\mathrm{H}$ & -6.49140200 & 3.19227000 & -0.31238000 \\
\hline $\mathrm{H}$ & -8.31209700 & 1.45260800 & -0.34635600 \\
\hline $\mathrm{H}$ & -7.80509400 & 1.80743400 & -2.01656700 \\
\hline $\mathrm{H}$ & -7.23703900 & 0.32805000 & -1.19700200 \\
\hline $\mathrm{H}$ & -5.58018000 & 2.13390800 & 1.35263000 \\
\hline $\mathrm{H}$ & -5.42676600 & 2.60927300 & -2.46046200 \\
\hline $\mathrm{H}$ & -5.02646700 & -0.01788400 & -1.27171500 \\
\hline $\mathrm{H}$ & -1.19674300 & 2.48619000 & 0.67114600 \\
\hline $\mathrm{H}$ & -2.07055200 & 4.02137300 & 0.38405900 \\
\hline $\mathrm{H}$ & -2.71671100 & 1.34887700 & -0.69256000 \\
\hline $\mathrm{H}$ & -1.88738700 & 0.38256600 & 4.49516600 \\
\hline $\mathrm{H}$ & -1.69846400 & 1.93354400 & 3.65062100 \\
\hline $\mathrm{H}$ & -3.63593700 & 1.41288300 & 2.18552300 \\
\hline $\mathrm{H}$ & -4.10080100 & 1.12730400 & 5.09763100 \\
\hline $\mathrm{H}$ & -6.39174200 & -3.41202200 & 0.36100100 \\
\hline $\mathrm{H}$ & -8.26743100 & -1.74589400 & 0.48711500 \\
\hline $\mathrm{H}$ & -7.66904800 & -2.09872400 & 2.12484600 \\
\hline $\mathrm{H}$ & -7.20160800 & -0.58998300 & 1.29882000 \\
\hline $\mathrm{H}$ & -5.54972700 & -2.31404500 & -1.34233700 \\
\hline $\mathrm{H}$ & -5.28863700 & -2.78367500 & 2.48903100 \\
\hline $\mathrm{H}$ & -5.02881300 & -0.14759500 & 1.23930400 \\
\hline $\mathrm{H}$ & -1.05013900 & -2.44832200 & -0.61497900 \\
\hline $\mathrm{H}$ & -1.86072500 & -4.02628100 & -0.43269300 \\
\hline $\mathrm{H}$ & -2.74721900 & -1.41849900 & 0.57879100 \\
\hline $\mathrm{H}$ & 2.96998900 & -4.73456900 & -0.55691400 \\
\hline $\mathrm{H}$ & 4.41112000 & -4.01352500 & -1.31827300 \\
\hline $\mathrm{H}$ & 2.82310000 & -3.89625300 & -2.11241500 \\
\hline
\end{tabular}

$\begin{array}{rrrr}\text { H } & 1.98383500 & -2.49268600 & -0.23102100 \\ \mathrm{H} & 4.35604700 & -1.47367300 & -1.59905900 \\ \mathrm{H} & -5.34370700 & -2.83827600 & -6.10339100 \\ \mathrm{H} & -4.90954500 & -4.32802900 & -5.22890900 \\ \mathrm{H} & -6.52090400 & -3.63462700 & -5.02103500 \\ \mathrm{H} & -1.86308800 & 2.60259700 & -2.60017200 \\ \mathrm{H} & -0.66598100 & 3.39527500 & -4.41683500 \\ \mathrm{H} & 0.16273500 & 4.56491300 & -3.34988000 \\ \mathrm{H} & 0.75379500 & 2.89098700 & -3.46454800 \\ \mathrm{H} & 7.70033200 & 3.94628300 & 0.65629400 \\ \mathrm{H} & 9.05639300 & 4.00822800 & -0.49059900 \\ \mathrm{H} & 8.08858800 & 2.52397700 & -0.31118600 \\ \mathrm{H} & 8.01033400 & 1.63940600 & 1.82800600 \\ \mathrm{H} & 9.10544200 & 0.79033300 & 3.73477700 \\ \mathrm{H} & 10.41897600 & 0.71366300 & 2.53414400 \\ \mathrm{H} & 10.29840500 & 2.10247800 & 3.61840200 \\ \mathrm{H} & -6.62361800 & 3.36639200 & 5.04875200 \\ \mathrm{H} & -5.33748300 & 2.73581600 & 6.11308800 \\ \mathrm{H} & -5.10749400 & 4.25356900 & 5.20853700 \\ \mathrm{H} & -1.82808900 & -2.71412100 & 2.64505500 \\ \mathrm{H} & -0.55932800 & -3.35365200 & 4.46224300 \\ \mathrm{H} & 0.23961100 & -4.57345500 & 3.43132200 \\ \mathrm{H} & 0.86020000 & -2.90804000 & 3.49004900 \\ \mathrm{H} & 8.08203900 & -1.84097700 & 0.62155600 \\ \mathrm{H} & 7.25549300 & -3.25133400 & -0.01402800 \\ \mathrm{H} & 8.78382000 & -3.47247900 & 0.87805600 \\ \mathrm{H} & 7.82112800 & -1.24136200 & -1.67251400 \\ \mathrm{H} & 8.71104800 & -0.87098600 & -3.79395300 \\ \mathrm{H} & 10.28814900 & -1.42894700 & -3.17531600 \\ \mathrm{H} & 9.13758000 & -2.60430300 & -3.82511800 \\ \mathrm{H} & 2.85644000 & 4.23308600 & -2.53987800 \\ \mathrm{H} & 4.63411100 & 4.21965200 & -2.75984700 \\ \mathrm{H} & 3.62614900 & 3.07999100 & -3.65873300 \\ \mathrm{H} & 3.64962900 & 0.68019700 & 0.09514700 \\ \mathrm{H} & 1.98500600 & 2.88220600 & -0.99351800 \\ \mathrm{H} & 3.82152900 & 2.59870500 & 1.69559100 \\ \mathrm{H} & 2.05129000 & 2.75440200 & 1.71731300 \\ \mathrm{H} & 2.82616500 & 1.29924900 & 2.40027800 \\ \mathrm{H} & 0.59321400 & -0.43573200 & 2.19460300 \\ \mathrm{H} & -0.25426500 & 0.40826600 & -0.70032200 \\ \mathrm{H} & 2.28575600 & -3.64770600 & 1.71077200 \\ \mathrm{H} & 3.72759900 & -2.61147600 & 3.82499400 \\ \mathrm{H} & 3.44851900 & -4.36378500 & 3.64860800 \\ \mathrm{H} & 4.94183200 & -3.61689200 & 3.01577500 \\ \mathrm{H} & 5.84875200 & 0.39597300 & -0.95084200 \\ \mathrm{H} & 5.83463600 & -0.89887400 & -0.08807500 \\ \mathrm{H} & 2.75580000 & -1.27924400 & -1.97755000 \\ \mathrm{O} & 0.75372500 & 6.70763700 & -0.72141100 \\ \mathrm{H} & 0.44235700 & 5.78759700 & -0.69467400 \\ \mathrm{H} & 1.68905000 & 6.62262000 & -0.49882000 \\ \mathrm{H} & & \end{array}$

Total electronic Energy (HF) : -3976.0452608

Sum of electronic and zero-point Energies: -3974.937897

Sum of electronic and thermal Free Energies: -3975.021597

\section{Transition state $3+$ constrained water}

$\begin{array}{lrrrr}\mathrm{C} & -1 & -5.53006800 & -3.37610000 & -5.17232500 \\ \mathrm{C} & -1 & -5.02020400 & -2.60953500 & -3.96581000 \\ \mathrm{O} & -1 & -5.26069700 & -2.99621600 & -2.82017200 \\ \mathrm{~N} & -1 & -4.30057200 & -1.50096300 & -4.18406700 \\ \mathrm{C} & -1 & -3.48669600 & -0.91713700 & -3.13416000 \\ \mathrm{C} & -1 & -3.83427200 & 0.54084300 & -2.88740100 \\ \mathrm{O} & -1 & -3.35625400 & 1.42046400 & -3.60138700 \\ \mathrm{C} & 0 & -1.99663000 & -1.05149000 & -3.46470500 \\ \mathrm{C} & 0 & -1.07609300 & -0.76633600 & -2.25623900 \\ \mathrm{O} & 0 & -1.58512500 & -0.11653100 & -1.29460100 \\ \mathrm{O} & 0 & 0.07787100 & -1.22997200 & -2.31086900 \\ \mathrm{~N} & -1 & -4.67475800 & 0.78491900 & -1.86547900 \\ \mathrm{C} & -1 & -5.08093600 & 2.13836800 & -1.57486900 \\ \mathrm{C} & -1 & -3.89519400 & 2.97482500 & -1.05475400 \\ \mathrm{O} & -1 & -3.98168400 & 4.21014400 & -1.05704900 \\ \mathrm{C} & -1 & -6.23022800 & 2.18944200 & -0.53937300 \\ \mathrm{C} & -1 & -7.46389500 & 1.44850300 & -1.08013600 \\ \mathrm{O} & -1 & -5.82411400 & 1.53387100 & 0.64360100 \\ \mathrm{~N} & -1 & -2.81553100 & 2.35776600 & -0.61870700 \\ \mathrm{C} & 0 & -1.65525000 & 3.04901600 & -0.12537900 \\ \mathrm{C} & 0 & -0.63496400 & 3.36243200 & -1.20130000 \\ \mathrm{O} & 0 & 0.45724100 & 3.87828200 & -0.90051700 \\ \mathrm{~N} & 0 & -0.98600900 & 3.10446600 & -2.45586100 \\ \mathrm{C} & -1 & -0.10453300 & 3.47264100 & -3.53415000 \\ \mathrm{C} & -1 & 8.57911800 & 3.22866900 & 0.11672200 \\ \mathrm{C} & -1 & 9.52377900 & 2.76090600 & 1.19350200 \\ \mathrm{O} & -1 & 10.69758400 & 3.10269700 & 1.27519100 \\ \mathrm{~N} & -1 & 8.91580500 & 1.93572800 & 2.02858200 \\ \mathrm{C} & -1 & 9.75295000 & 1.23316200 & 2.94752700 \\ \mathrm{C} & -1 & -5.46989200 & 3.32487800 & 5.13345500 \\ \mathrm{C} & -1 & -4.93515000 & 2.60233200 & 3.91524900 \\ \mathrm{O} & -1 & -5.17292300 & 3.04969100 & 2.79486900 \\ \mathrm{~N} & -1 & -4.22093200 & 1.50012500 & 4.11838500 \\ \mathrm{C} & -1 & -3.44762600 & 0.86408300 & 3.06169900 \\ \mathrm{C} & -1 & -3.81879000 & -0.59011800 & 2.82382100 \\ \mathrm{O} & -1 & -3.34343400 & -1.50636500 & 3.54042700 \\ \mathrm{C} & 0 & -1.99872200 & 0.92179500 & 3.53857600 \\ \mathrm{C} & 0 & -1.03767400 & 0.23588800 & 2.60678000 \\ & & & & \end{array}$




$\begin{array}{rrrrr}\text { H } & 0 & 8.64064500 & -1.09303600 & -3.90252800 \\ \mathrm{H} & 0 & 10.24403600 & -1.54793200 & -3.26842100 \\ \mathrm{H} & \mathrm{O} & 9.15026700 & -2.80385900 & -3.86431500 \\ \mathrm{H} & 0 & 2.90155700 & 4.12995900 & -2.59601100 \\ \mathrm{H} & 0 & 4.67142100 & 4.12811000 & -2.86374800 \\ \mathrm{H} & 0 & 3.65074900 & 2.98393100 & -3.74113600 \\ \mathrm{H} & 0 & 3.98607300 & 0.97311300 & 0.40065500 \\ \mathrm{H} & 0 & 2.09095100 & 2.77217900 & -1.02740300 \\ \mathrm{H} & 0 & 3.65323700 & 3.14001700 & 1.58219600 \\ \mathrm{H} & 0 & 1.89027400 & 2.95778400 & 1.44562300 \\ \mathrm{H} & 0 & 2.81964100 & 1.83867700 & 2.47675300 \\ \mathrm{H} & 0 & 0.81979100 & -0.19881700 & 2.40274800 \\ \mathrm{H} & 0 & 0.06660800 & 0.18819200 & -0.22838200 \\ \mathrm{H} & 0 & 2.21165600 & -3.69378500 & 1.76227400 \\ \mathrm{H} & 0 & 3.41080700 & -3.27529200 & 3.98258600 \\ \mathrm{H} & 0 & 4.06692400 & -4.70664900 & 3.14117500 \\ \mathrm{H} & 0 & 4.88314600 & -3.12707300 & 2.99008200 \\ \mathrm{H} & 0 & 6.02008800 & 0.44602500 & -0.90980000 \\ \mathrm{H} & 0 & 5.88903200 & -0.84777100 & -0.05867500 \\ \mathrm{H} & 0 & 2.05646600 & -0.90434300 & -1.67689000 \\ \mathrm{O} & 0 & 1.03907300 & 6.51560100 & -0.69719800 \\ \mathrm{H} & 0 & 0.67428900 & 5.61954100 & -0.78678500 \\ \mathrm{H} & 0 & 1.80986400 & 6.37659500 & -0.13312100\end{array}$
Total electronic Energy (HF) : - 3976.038228

Sum of electronic and zero-point Energies: -3974.929787 Sum of electronic and thermal Free Energies: -3975.008497 Imaginary frequency: -144.74

\section{Extended model system: product complex}

\begin{tabular}{|c|c|c|c|}
\hline \\
\hline & \\
\hline \multirow{2}{*}{\multicolumn{4}{|c|}{$\begin{array}{lrrr}\text { C } & 11.98163800 & -4.66441100 & -0.91671200 \\
\text { C } & 10.58915100 & -4.26480500 & -1.38754000 \\
0 & 9.64267500 & -4.99689300 & -1.16574100\end{array}$}} \\
\hline & & & \\
\hline $\mathrm{N}$ & 10.42315700 & -3.11685300 & -2.03559700 \\
\hline $\mathrm{C}$ & 9.15917200 & -2.73679500 & -2.62516400 \\
\hline $\mathrm{C}$ & 8.71873200 & -3.82925400 & -3.59758100 \\
\hline 0 & 9.55015300 & -4.21416600 & -4.42349000 \\
\hline $\mathrm{C}$ & 9.28475400 & -1.40013600 & -3.32282200 \\
\hline $\mathrm{C}$ & 9.60405500 & -0.22095500 & -2.40069500 \\
\hline $\mathrm{C}$ & 9.54646200 & 1.13127400 & -3.12142500 \\
\hline $\mathrm{N}$ & 9.92331700 & 2.18266900 & -2.19819900 \\
\hline $\mathrm{C}$ & 10.42190000 & 3.36652500 & -2.49453400 \\
\hline $\mathrm{N}$ & 10.63282800 & 3.70886800 & -3.75500400 \\
\hline $\mathrm{N}$ & 10.72006500 & 4.23031400 & -1.53622200 \\
\hline $\mathrm{N}$ & 7.46913600 & -4.28323100 & -3.47841800 \\
\hline $\mathrm{C}$ & 6.95452000 & -5.29351700 & -4.42912100 \\
\hline $\mathrm{C}$ & 6.54005400 & -4.72019400 & -5.78725700 \\
\hline 0 & 5.37290500 & -4.49547200 & -6.15534700 \\
\hline $\mathrm{C}$ & 5.73407200 & -5.83453500 & -3.65599800 \\
\hline C & 5.24096300 & -4.66475000 & -2.86610300 \\
\hline $\mathrm{C}$ & 6.47967000 & -3.88586400 & -2.46193000 \\
\hline $\mathrm{N}$ & 2.76335600 & -3.21914000 & -6.18925500 \\
\hline $\mathrm{C}$ & 1.57103000 & -3.88679700 & -5.66060700 \\
\hline $\mathrm{C}$ & 1.21042400 & -3.36256000 & -4.28276300 \\
\hline 0 & 1.93106100 & -3.58974500 & -3.30865800 \\
\hline $\mathrm{C}$ & 1.83994900 & -5.40040500 & -5.56924100 \\
\hline $\mathrm{N}$ & -3.81804100 & 0.68839200 & -1.10984700 \\
\hline C & -4.46084200 & 1.56049100 & -2.05934200 \\
\hline $\mathrm{C}$ & -5.80091700 & 0.98452400 & -2.48650200 \\
\hline 0 & -5.87551000 & -0.15527400 & -2.93662800 \\
\hline $\mathrm{C}$ & -3.62324600 & 1.74649700 & -3.30659600 \\
\hline $\mathrm{N}$ & -1.31867500 & 10.64466000 & 2.66045600 \\
\hline $\mathrm{C}$ & -0.37045100 & 11.49225300 & 3.30951300 \\
\hline C & -12.13362700 & -5.02414400 & 1.61793400 \\
\hline $\mathrm{C}$ & -10.75634100 & -4.50501300 & 2.01769800 \\
\hline 0 & -9.75311800 & -5.20928600 & 1.75609400 \\
\hline $\mathrm{N}$ & -10.68145200 & -3.31857500 & 2.63196400 \\
\hline $\mathrm{C}$ & -9.36971100 & -2.90297600 & 3.11842200 \\
\hline $\mathrm{C}$ & -8.83451800 & -3.90887100 & 4.12170300 \\
\hline 0 & -9.60142400 & -4.35454400 & 4.99940500 \\
\hline $\mathrm{C}$ & -9.46826800 & -1.53190500 & 3.74642100 \\
\hline $\mathrm{C}$ & -9.78292600 & -0.39468800 & 2.79540900 \\
\hline $\mathrm{C}$ & -9.81110100 & 0.92557700 & 3.57217300 \\
\hline $\mathrm{N}$ & -10.17806800 & 1.98455300 & 2.62865300 \\
\hline $\mathrm{C}$ & -10.70086400 & 3.16769500 & 2.94626800 \\
\hline $\mathrm{N}$ & -10.92585200 & 3.45504800 & 4.22495100 \\
\hline $\mathrm{N}$ & -10.97676600 & 4.01580500 & 1.96115800 \\
\hline $\mathrm{N}$ & -7.56195200 & -4.27522900 & 4.02131700 \\
\hline $\mathrm{C}$ & -6.99883600 & -5.20800900 & 5.01119400 \\
\hline $\mathrm{C}$ & -6.66551500 & -4.57615000 & 6.36828100 \\
\hline 0 & -5.51109500 & -4.33111900 & 6.72314300 \\
\hline $\mathrm{C}$ & -5.69083200 & -5.68107500 & 4.32866500 \\
\hline C & -5.29081600 & -4.58771200 & 3.40912900 \\
\hline $\mathrm{C}$ & -6.56443800 & -3.88108600 & 3.02309700 \\
\hline $\mathrm{N}$ & 3.70422000 & 0.76092500 & 1.55291800 \\
\hline $\mathrm{C}$ & 4.36158000 & 1.67136500 & 2.46903000 \\
\hline $\mathrm{C}$ & 5.68362500 & 1.10362200 & 2.93830100 \\
\hline 0 & 5.77155700 & -0.02084600 & 3.41690800 \\
\hline $\mathrm{C}$ & 3.50099400 & 1.88572800 & 3.71643100 \\
\hline $\mathrm{N}$ & 6.72864600 & 1.89874400 & 2.79745700 \\
\hline $\mathrm{C}$ & 8.03344000 & 1.44869300 & 3.29727800 \\
\hline $\mathrm{C}$ & 8.04885900 & 1.30879900 & 4.81408200 \\
\hline 0 & 8.67022800 & 0.43426600 & 5.38408000 \\
\hline $\mathrm{C}$ & 9.14692500 & 2.40675100 & 2.91968700 \\
\hline
\end{tabular}




\begin{tabular}{|c|c|c|c|}
\hline C & 9.40858600 & 2.58497900 & 1.45465400 \\
\hline 0 & 8.87650500 & 1.82727700 & 0.62050100 \\
\hline 0 & 10.17210900 & 3.51424700 & 1.12068200 \\
\hline $\mathrm{N}$ & 1.15916700 & 10.27949200 & -2.38421800 \\
\hline C & 0.63553400 & 10.87062800 & -3.62399200 \\
\hline $\mathrm{N}$ & 7.57439700 & -4.46953000 & -6.58519100 \\
\hline $\mathrm{C}$ & 7.47183900 & -3.94432300 & -7.92453200 \\
\hline $\mathrm{C}$ & 3.95759000 & -1.31370600 & -7.06134000 \\
\hline C & 2.65908300 & -1.99442900 & -6.69498100 \\
\hline 0 & 1.60149700 & -1.38827500 & -6.88188800 \\
\hline $\mathrm{N}$ & -7.72665300 & -4.31952200 & 7.12100700 \\
\hline C & -7.65704400 & -3.74553300 & 8.43816800 \\
\hline $\mathrm{N}$ & -7.45160500 & 2.14293600 & -5.00421600 \\
\hline & -7.49073500 & 2.41670200 & -6.41862200 \\
\hline N & 6.13796600 & -2.05337800 & 5.29860300 \\
\hline $\mathrm{C}$ & 6.95944300 & -3.24562800 & 5.08266400 \\
\hline c & 7.95576200 & -3.50403600 & 6.20149200 \\
\hline 0 & 8.33720600 & -4.65582100 & 6.44653600 \\
\hline c & 7.72099700 & -3.13135900 & 3.73734200 \\
\hline C & 6.81605000 & -3.24258500 & 2.55013400 \\
\hline c & 7.49084600 & -3.36096100 & 1.19038300 \\
\hline N & 8.29697200 & -2.12310900 & 1.00684000 \\
\hline c & 9.58593000 & -2.02066000 & 1.24205700 \\
\hline $\mathrm{N}$ & 10.34483100 & -3.02120500 & 1.67988200 \\
\hline $\mathrm{N}$ & 10.20257300 & -0.84635100 & 1.03796100 \\
\hline $\mathrm{N}$ & 7.34071300 & 2.20113900 & 5.47421600 \\
\hline & 7.37899300 & 2.47664600 & 6.88478300 \\
\hline $\mathrm{N}$ & -6.85341500 & 1.77057100 & -2.34610100 \\
\hline C & -8.13879600 & 1.32298300 & -2.84316000 \\
\hline c & -8.17456200 & 1.23430800 & -4.36431700 \\
\hline 0 & -8.83503600 & 0.37418800 & -4.93876200 \\
\hline & -9.21009300 & 2.28599300 & -2.37064800 \\
\hline c & -9.43532500 & 2.42324100 & -0.88059000 \\
\hline o & -9.08179200 & 1.50505300 & -0.11530700 \\
\hline & -9.98778700 & 3.50924900 & -0.52462100 \\
\hline N & -6.19551700 & -2.28674900 & -4.74913300 \\
\hline C & -6.99138000 & -3.46570200 & -4.46133500 \\
\hline c & -7.99814800 & -3.77099300 & -5.56317600 \\
\hline o & -8.35758500 & -4.937777000 & -5.76289500 \\
\hline C & -7.73771300 & -3.28696700 & -3.12937400 \\
\hline c & -6.84240400 & -3.33805500 & -1.90999100 \\
\hline c & -7.58022300 & -3.32747500 & -0.59416100 \\
\hline N & -8.45012400 & -2.14964800 & -0.46994200 \\
\hline c & -9.75296300 & -2.12091200 & -0.67615500 \\
\hline $\mathrm{N}$ & -10.48038000 & -3.18569000 & -1.03675400 \\
\hline $\mathrm{N}$ & -10.41333700 & -0.99579200 & -0.52944700 \\
\hline $\mathrm{N}$ & -8.47510600 & -2.75857200 & -6.29480700 \\
\hline c & -9.50624700 & -3.00006200 & -7.29925200 \\
\hline $\mathrm{N}$ & 8.40848300 & -2.47793100 & 6.90944000 \\
\hline c & 9.37784100 & -2.71479700 & 7.96725700 \\
\hline c & -1.64104600 & -3.70306500 & 6.20383200 \\
\hline c & -1.32929900 & -3.18290900 & 4.81663700 \\
\hline o & -2.09835100 & -3.43365000 & 3.89056900 \\
\hline c & -3.13503900 & 10.28590400 & 1.24196100 \\
\hline C & -2.36223300 & 11.24328400 & 2.11195500 \\
\hline 0 & -2.66630800 & 12.41797700 & 2.28177800 \\
\hline c & -4.48231200 & -1.05769000 & -5.89905300 \\
\hline C & -5.43354800 & -2.24686200 & -5.83355500 \\
\hline o & -5.46345400 & -3.07479000 & -6.72729100 \\
\hline c & 2.76466100 & 9.91948300 & -0.66154800 \\
\hline C & 2.28634700 & 10.70685700 & -1.88230600 \\
\hline b & 2.93071900 & 11.65256600 & -2.32543500 \\
\hline C & 4.36033200 & -0.83935000 & 6.36410300 \\
\hline C & 5.33146400 & -1.99856200 & 6.35137800 \\
\hline 0 & 5.36094500 & -2.83501500 & 7.26916900 \\
\hline o & -0.14503000 & 8.04324100 & -0.09166500 \\
\hline N & 0.08599800 & -2.64505300 & -4.15812700 \\
\hline$C$ & -0.14948000 & -1.81864800 & -2.98881200 \\
\hline C & -1.46026200 & -2.16260700 & -2.30229200 \\
\hline b & -2.51816700 & -1.69278100 & -2.71702800 \\
\hline C & -0.17598900 & -0.37228000 & -3.48754500 \\
\hline$C$ & -0.31835500 & 0.71327800 & -2.43160300 \\
\hline 0 & -0.48460900 & 0.32507200 & -1.21157600 \\
\hline 0 & -0.25989300 & 1.88732000 & -2.80404500 \\
\hline $\mathrm{N}$ & -1.37592500 & -2.99110900 & -1.24540200 \\
\hline C & -2.57348200 & -3.39329600 & -0.54865500 \\
\hline C & -3.21356200 & -2.20104700 & 0.18974000 \\
\hline 0 & -4.38957400 & -2.28712100 & 0.56812200 \\
\hline c & -2.30034800 & -4.53026800 & 0.46547400 \\
\hline C & -1.75737500 & -5.77052500 & -0.26282900 \\
\hline 0 & -1.31443000 & -4.11061200 & 1.38519000 \\
\hline $\mathrm{N}$ & -2.49576500 & -1.11674200 & 0.40322900 \\
\hline C & -3.03525500 & 0.02937800 & 1.08852600 \\
\hline c & -3.78412700 & 0.99660100 & 0.18761300 \\
\hline 0 & -4.29657200 & 2.01350300 & 0.67994000 \\
\hline N & -0.22027300 & -2.46689100 & 4.66332200 \\
\hline $\mathrm{C}$ & 0.05809000 & -1.70635200 & 3.45379700 \\
\hline 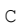 & 1.37007100 & -2.08070700 & 2.78484000 \\
\hline 0 & 2.46061400 & -1.59751000 & 3.18007900 \\
\hline${ }^{2}$ & 0.08857800 & -0.21807900 & 3.79737300 \\
\hline$C$ & 0.28846100 & 0.60903000 & 2.55332100 \\
\hline & 0.28479800 & 0.12138600 & 1.43576700 \\
\hline & 0.43813100 & 1.89269400 & 2.79197100 \\
\hline & 1.31642200 & -2.94541500 & 1.75972800 \\
\hline & 2.55008300 & -3.31145400 & 1.07281000 \\
\hline
\end{tabular}

\begin{tabular}{|c|c|c|}
\hline 3.17249000 & -2.16807900 & 0.25524900 \\
\hline 4.34703700 & -2.27806600 & -0.15516400 \\
\hline 2.30530800 & -4.50872800 & 0.12722300 \\
\hline 1.81096700 & -5.69973200 & 0.93332100 \\
\hline 1.30291900 & -4.13973300 & -0.82403800 \\
\hline 2.40645100 & -1.09411500 & 0.02354500 \\
\hline 2.86638500 & 0.08555600 & -0.64902800 \\
\hline 3.63709700 & 1.03375000 & 0.24806500 \\
\hline 4.14506600 & 2.04242400 & -0.26037600 \\
\hline 6.57127200 & -0.70977900 & -0.63413100 \\
\hline 6.75771800 & 2.03382300 & -0.98375100 \\
\hline-6.67586600 & -0.71593800 & 1.09794700 \\
\hline-6.94303300 & 1.91887100 & 1.70525500 \\
\hline-7.55135700 & 5.13386700 & 1.25552100 \\
\hline-6.55778800 & 4.82988400 & 0.16529600 \\
\hline-6.64586900 & 3.78381600 & -0.47756100 \\
\hline-5.58703800 & 5.73753600 & -0.07165800 \\
\hline-4.60933500 & 5.59313100 & -1.13593800 \\
\hline-3.17517100 & 5.67940700 & -0.60440000 \\
\hline-2.52994000 & 6.70266900 & -0.81403400 \\
\hline-1.45344600 & 4.56262800 & 0.75019200 \\
\hline 2.12555000 & 5.20371200 & -1.17073800 \\
\hline 1.87580400 & 6.35043200 & -0.82436900 \\
\hline-2.75003400 & 4.61651800 & 0.09658400 \\
\hline-0.65055500 & 3.34452000 & 0.31129900 \\
\hline-1.63015300 & 4.49368200 & 2.27261200 \\
\hline 0.55850800 & 3.26325100 & 0.57632500 \\
\hline-1.36868500 & 2.37703500 & -0.15834600 \\
\hline-0.13251200 & 4.75645300 & -1.95381500 \\
\hline 1.26862900 & 4.41902800 & -2.15529600 \\
\hline 1.77719000 & 4.65810000 & -3.58315300 \\
\hline 3.25921700 & 4.56112200 & -0.76955400 \\
\hline 3.96941000 & 4.98739800 & 0.41700500 \\
\hline 5.46734000 & 4.71226300 & 0.33026700 \\
\hline 6.10069400 & 4.26765700 & 1.29152100 \\
\hline 6.03389600 & 5.03515300 & -0.84334800 \\
\hline 7.45618200 & 4.93561000 & -1.10471800 \\
\hline 1.92942500 & 9.55120800 & -0.05821400 \\
\hline 3.34137700 & 9.05037000 & -0.99446600 \\
\hline 3.41052200 & 10.56362600 & -0.06465700 \\
\hline 0.63205300 & 9.56271700 & -1.89503700 \\
\hline 0.29711300 & 11.89650000 & -3.45385500 \\
\hline 1.42761900 & 10.89274200 & -4.37511900 \\
\hline-4.03759600 & 10.77015500 & 0.86808200 \\
\hline-2.52188300 & 9.96631700 & 0.39255500 \\
\hline-3.40826700 & 9.38857800 & 1.80606300 \\
\hline-0.96578400 & 7.60072400 & -0.36794300 \\
\hline 0.56347900 & 7.42278700 & -0.33572900 \\
\hline-0.88576400 & 12.10708100 & 4.04884700 \\
\hline 0.38364000 & 10.88659200 & 3.81606300 \\
\hline 0.12860000 & 12.16918900 & 2.60584500 \\
\hline-0.98804400 & 9.78542500 & 2.23805800 \\
\hline-5.57306600 & 6.59210200 & 0.46824700 \\
\hline-7.50415500 & 4.31358300 & 1.97850100 \\
\hline-7.38081300 & 6.09104100 & 1.75226200 \\
\hline-8.54282500 & 5.11004100 & 0.79350500 \\
\hline-4.72214000 & 6.39610800 & -1.86799200 \\
\hline-4.80274000 & 4.63320900 & -1.61766800 \\
\hline-3.35281700 & 3.79811100 & 0.19992500 \\
\hline-0.88953100 & 5.45194100 & 0.46706400 \\
\hline-0.31344100 & 5.71843100 & -2.23800400 \\
\hline 1.67161800 & 5.71470200 & -3.85263000 \\
\hline 2.83087500 & 4.37762600 & -3.68934000 \\
\hline 1.18736600 & 4.05026100 & -4.27487100 \\
\hline 1.36002500 & 3.35454800 & -1.92123800 \\
\hline 3.28278700 & 3.55698200 & -0.91822200 \\
\hline 3.80707000 & 6.06358700 & 0.53600100 \\
\hline 3.58665100 & 4.48480900 & 1.31179400 \\
\hline 7.83939200 & 5.90018500 & -1.45090800 \\
\hline 7.95664200 & 4.66609000 & -0.17198600 \\
\hline 7.65451900 & 4.15637300 & -1.84556700 \\
\hline 5.40680200 & 5.33044600 & -1.58120800 \\
\hline-0.19819200 & 10.26420400 & -3.97916400 \\
\hline-0.68170300 & 4.12592200 & -2.53616800 \\
\hline-0.83488600 & 1.51469400 & -0.53469800 \\
\hline 0.58387800 & 2.37403000 & 1.91623100 \\
\hline 3.54642600 & -1.04849400 & 5.65950700 \\
\hline 4.84452000 & 0.08707500 & 6.03665100 \\
\hline 6.08692300 & -1.33961000 & 4.57992700 \\
\hline 6.32305700 & -4.13769900 & 5.07473100 \\
\hline 8.95014900 & -3.38638800 & 8.71525900 \\
\hline 10.28208300 & -3.18399600 & 7.56850700 \\
\hline 9.63097400 & -1.76013400 & 8.42915700 \\
\hline 8.16253300 & -1.52994800 & 6.64753600 \\
\hline 8.27706200 & -2.18248100 & 3.74414500 \\
\hline 8.45321400 & -3.95077100 & 3.74506700 \\
\hline 6.16948800 & -4.12227800 & 2.67878900 \\
\hline 6.14234900 & -2.37638200 & 2.50195100 \\
\hline 6.73809100 & -3.34876300 & 0.39969600 \\
\hline 8.11438000 & -4.25031500 & 1.05427500 \\
\hline 7.79198200 & -1.36853800 & 0.49990400 \\
\hline 9.66656300 & 0.01371800 & 0.86809600 \\
\hline 11.19308000 & -0.75543800 & 1.20063700 \\
\hline 9.92266600 & -3.91401800 & 1.89189800 \\
\hline 11.19491700 & -2.81190800 & 2.18201100 \\
\hline
\end{tabular}




\begin{tabular}{|c|c|c|c|}
\hline $\mathrm{H}$ & 5.67289600 & -1.06662800 & -0.49098700 \\
\hline $\mathrm{H}$ & 6.52607700 & 0.26197800 & -0.79846300 \\
\hline $\mathrm{H}$ & 5.85844200 & 2.30457000 & -0.72675100 \\
\hline $\mathrm{H}$ & 7.32392900 & 2.14958600 & -0.19574000 \\
\hline $\mathrm{H}$ & 10.54858400 & 3.97372600 & -0.53382200 \\
\hline $\mathrm{H}$ & 10.93259400 & 5.19005400 & -1.76164400 \\
\hline $\mathrm{H}$ & 9.57456200 & 2.07641200 & -1.22820300 \\
\hline $\mathrm{H}$ & 11.14911700 & 4.54286400 & -3.98859000 \\
\hline $\mathrm{H}$ & 10.30416200 & 3.14078600 & -4.51956900 \\
\hline $\mathrm{H}$ & 8.53431600 & 1.30738100 & -3.50696600 \\
\hline $\mathrm{H}$ & 10.24935000 & 1.12121600 & -3.96418400 \\
\hline $\mathrm{H}$ & 8.87051600 & -0.20398300 & -1.58288300 \\
\hline $\mathrm{H}$ & 10.59564100 & -0.33400800 & -1.94665700 \\
\hline $\mathrm{H}$ & 10.03396800 & -1.49192300 & -4.12190500 \\
\hline $\mathrm{H}$ & 8.32650000 & -1.20370400 & -3.82260700 \\
\hline $\mathrm{H}$ & 8.43663200 & -2.63321900 & -1.80685600 \\
\hline $\mathrm{H}$ & 11.24942500 & -2.66189700 & -2.40299000 \\
\hline $\mathrm{H}$ & -1.01922400 & -0.25197600 & -4.17822600 \\
\hline $\mathrm{H}$ & 0.73013100 & -0.17465100 & -4.06931200 \\
\hline $\mathrm{H}$ & 6.31728600 & -2.80457800 & -2.43506700 \\
\hline $\mathrm{H}$ & 6.86143300 & -4.20913700 & -1.48761200 \\
\hline $\mathrm{H}$ & 4.58815200 & -4.04082300 & -3.48616500 \\
\hline $\mathrm{H}$ & 4.65609300 & -4.95924700 & -1.99254200 \\
\hline $\mathrm{H}$ & 3.30699700 & -3.57400700 & 1.81872300 \\
\hline $\mathrm{H}$ & 1.68267300 & -3.94386000 & -1.71071100 \\
\hline $\mathrm{H}$ & 0.83656700 & -5.47930900 & 1.37860400 \\
\hline $\mathrm{H}$ & 1.69283900 & -6.56812400 & 0.27968500 \\
\hline $\mathrm{H}$ & 2.51779600 & -5.95262800 & 1.72901900 \\
\hline $\mathrm{H}$ & 3.25264500 & -4.72652000 & -0.37456800 \\
\hline $\mathrm{H}$ & -2.44680900 & -6.10142300 & -1.04474800 \\
\hline $\mathrm{H}$ & -0.78680500 & -5.54228000 & -0.71286000 \\
\hline $\mathrm{H}$ & -1.61944800 & -6.58242600 & 0.45580100 \\
\hline $\mathrm{H}$ & -1.71411900 & -3.87068700 & 2.25100600 \\
\hline $\mathrm{H}$ & -3.24494800 & -4.74481100 & 0.97407900 \\
\hline $\mathrm{H}$ & -8.34745200 & -4.26214000 & 9.10886300 \\
\hline $\mathrm{H}$ & -6.63787500 & -3.86136300 & 8.80814100 \\
\hline $\mathrm{H}$ & -7.90593700 & -2.67821700 & 8.43010600 \\
\hline $\mathrm{H}$ & -8.63640900 & -4.48355500 & 6.69641500 \\
\hline $\mathrm{H}$ & -7.71767300 & -6.01823900 & 5.16327000 \\
\hline $\mathrm{H}$ & -5.91414400 & -6.59796500 & 3.77230600 \\
\hline $\mathrm{H}$ & -4.93204200 & -5.90739900 & 5.07671000 \\
\hline $\mathrm{H}$ & -4.61528900 & -3.89548500 & 3.91999900 \\
\hline $\mathrm{H}$ & -4.76317300 & -4.94903400 & 2.52424500 \\
\hline $\mathrm{H}$ & -6.45060900 & -2.79305200 & 2.96258800 \\
\hline $\mathrm{H}$ & -6.93083100 & -4.22904500 & 2.04977100 \\
\hline $\mathrm{H}$ & -12.92308700 & -4.27210800 & 1.69737100 \\
\hline $\mathrm{H}$ & -12.38094600 & -5.87401700 & 2.25886400 \\
\hline $\mathrm{H}$ & -11.49712400 & -2.98805800 & 3.13178400 \\
\hline $\mathrm{H}$ & 8.96234000 & 3.40108000 & 3.34267500 \\
\hline $\mathrm{H}$ & 10.07326100 & 2.05414000 & 3.38896600 \\
\hline $\mathrm{H}$ & 6.37040300 & 2.47636400 & 7.30599500 \\
\hline $\mathrm{H}$ & 7.96217900 & 1.69165000 & 7.36750300 \\
\hline $\mathrm{H}$ & 7.85090500 & 3.44379200 & 7.08140900 \\
\hline $\mathrm{H}$ & 6.84250900 & 2.87782200 & 4.90856300 \\
\hline $\mathrm{H}$ & 8.22960000 & 0.44780600 & 2.89833600 \\
\hline $\mathrm{H}$ & 6.64323800 & 2.77102200 & 2.27087000 \\
\hline $\mathrm{H}$ & 2.58036100 & 2.41612200 & 3.47364100 \\
\hline $\mathrm{H}$ & 3.25457000 & 0.91183000 & 4.15323100 \\
\hline $\mathrm{H}$ & 4.05716500 & 2.46340700 & 4.46251900 \\
\hline $\mathrm{H}$ & 4.53224000 & 2.60854700 & 1.93854800 \\
\hline $\mathrm{H}$ & 3.50821700 & -0.17063200 & -1.49700500 \\
\hline $\mathrm{H}$ & 1.98997700 & 0.63332500 & -1.01199200 \\
\hline $\mathrm{H}$ & 12.66808800 & -3.81820900 & -0.82442600 \\
\hline $\mathrm{H}$ & 7.50617100 & -2.84905100 & -7.93490700 \\
\hline $\mathrm{H}$ & 8.29395600 & -4.33183700 & -8.52775600 \\
\hline $\mathrm{H}$ & 6.52180700 & -4.26375200 & -8.35531100 \\
\hline $\mathrm{H}$ & 8.49982700 & -4.57761700 & -6.17396000 \\
\hline $\mathrm{H}$ & 7.73351700 & -6.04402200 & -4.58679700 \\
\hline $\mathrm{H}$ & 4.98381600 & -6.24000100 & -4.33668200 \\
\hline $\mathrm{H}$ & 6.07609200 & -6.63891100 & -2.99648500 \\
\hline $\mathrm{H}$ & 3.31404200 & -0.10301500 & 1.93362900 \\
\hline $\mathrm{H}$ & -0.84992600 & 0.08199300 & 4.27572100 \\
\hline $\mathrm{H}$ & 0.90401000 & 0.00048300 & 4.49508700 \\
\hline $\mathrm{H}$ & -0.76419600 & -1.90967000 & 2.76213900 \\
\hline $\mathrm{H}$ & 0.41975200 & -3.31777000 & 1.42984500 \\
\hline $\mathrm{H}$ & -3.33059400 & -3.71865200 & -1.27038400 \\
\hline $\mathrm{H}$ & -0.46801500 & -3.36033700 & -0.95257800 \\
\hline $\mathrm{H}$ & -1.56865000 & -1.00884500 & -0.01228100 \\
\hline $\mathrm{H}$ & -3.71419600 & -0.29519700 & 1.88189500 \\
\hline $\mathrm{H}$ & -2.21520400 & 0.60008000 & 1.52960300 \\
\hline $\mathrm{H}$ & 0.68319600 & -1.97993900 & -2.29962300 \\
\hline $\mathrm{H}$ & -0.35697000 & -2.34131100 & -5.02019300 \\
\hline $\mathrm{H}$ & 0.75209700 & -3.68249300 & -6.35674900 \\
\hline $\mathrm{H}$ & 0.95048300 & -5.92915500 & -5.21813000 \\
\hline $\mathrm{H}$ & 2.12127700 & -5.78372200 & -6.55199900 \\
\hline $\mathrm{H}$ & 2.65562500 & -5.58027000 & -4.86334600 \\
\hline $\mathrm{H}$ & 3.67218300 & -3.65722800 & -6.04871000 \\
\hline $\mathrm{H}$ & -3.64268300 & -1.23285600 & -5.21622200 \\
\hline $\mathrm{H}$ & -4.97753300 & -0.13384100 & -5.58056900 \\
\hline $\mathrm{H}$ & -6.09381900 & -1.56609700 & -4.04260500 \\
\hline $\mathrm{H}$ & -6.35526300 & -4.35805800 & -4.41995900 \\
\hline $\mathrm{H}$ & -9.78437500 & -2.04548500 & -7.74605700 \\
\hline $\mathrm{H}$ & -9.12166100 & -3.67182200 & -8.07021600 \\
\hline $\mathrm{H}$ & -10.38566400 & -3.46816300 & -6.84701000 \\
\hline $\mathrm{H}$ & -8.22002200 & -1.80712100 & -6.05789000 \\
\hline
\end{tabular}

\begin{tabular}{|c|c|c|c|}
\hline $\mathrm{H}$ & -8.28840300 & -2.33613500 & -3.17440200 \\
\hline $\mathrm{H}$ & -8.47752000 & -4.09863200 & -3.08451600 \\
\hline $\mathrm{H}$ & -6.23072700 & -4.24903200 & -1.94242300 \\
\hline $\mathrm{H}$ & -8.16313400 & -4.23869200 & -0.41432200 \\
\hline $\mathrm{H}$ & -6.85900000 & -3.24649000 & 0.22314700 \\
\hline $\mathrm{H}$ & -7.98105600 & -1.34109900 & -0.02231000 \\
\hline $\mathrm{H}$ & -8.70844200 & -2.85344800 & 2.24533000 \\
\hline $\mathrm{H}$ & -10.20662600 & -1.58099200 & 4.56051700 \\
\hline $\mathrm{H}$ & -8.50429900 & -1.32313600 & 4.23060100 \\
\hline $\mathrm{H}$ & -10.74917800 & -0.54025200 & 2.29966700 \\
\hline $\mathrm{H}$ & -9.00704400 & -0.33480400 & 2.02069600 \\
\hline $\mathrm{H}$ & -10.56949200 & 0.86498200 & 4.36210900 \\
\hline $\mathrm{H}$ & -8.83070300 & 1.13043200 & 4.01888100 \\
\hline $\mathrm{H}$ & -9.81135200 & 1.87741200 & 1.66838000 \\
\hline $\mathrm{H}$ & -11.41136300 & 4.29674600 & 4.49461300 \\
\hline $\mathrm{H}$ & -10.61214400 & 2.84244600 & 4.96032800 \\
\hline $\mathrm{H}$ & -11.24201500 & 4.96616000 & 2.17388600 \\
\hline $\mathrm{H}$ & -10.64455000 & 3.79251400 & 0.98354000 \\
\hline $\mathrm{H}$ & -9.01390300 & 3.29154600 & -2.75856700 \\
\hline $\mathrm{H}$ & -10.16626300 & 1.98051400 & -2.81596900 \\
\hline $\mathrm{H}$ & -6.78714600 & 2.61311700 & -1.77756600 \\
\hline $\mathrm{H}$ & -8.33460500 & 0.30938500 & -2.47630200 \\
\hline $\mathrm{H}$ & -7.94475200 & 3.39222500 & -6.61610400 \\
\hline $\mathrm{H}$ & -6.48470800 & 2.39349300 & -6.84526400 \\
\hline $\mathrm{H}$ & -8.09322900 & 1.64020000 & -6.89100200 \\
\hline $\mathrm{H}$ & -6.92674900 & 2.78615100 & -4.42619400 \\
\hline $\mathrm{H}$ & -7.49499700 & 2.04120400 & 0.90812800 \\
\hline $\mathrm{H}$ & -6.05275000 & 2.20061500 & 1.41354000 \\
\hline $\mathrm{H}$ & -6.63260900 & 0.22438300 & 1.38140500 \\
\hline $\mathrm{H}$ & -5.77951000 & -1.06099700 & 0.92233300 \\
\hline $\mathrm{H}$ & -11.42000000 & -0.98550000 & -0.57636600 \\
\hline $\mathrm{H}$ & -9.91943700 & -0.10237800 & -0.38703200 \\
\hline $\mathrm{H}$ & -11.31257400 & -3.03240100 & -1.58713700 \\
\hline $\mathrm{H}$ & -10.01893600 & -4.07148500 & -1.19090400 \\
\hline $\mathrm{H}$ & -12.07262800 & -5.38706500 & 0.59116800 \\
\hline $\mathrm{H}$ & -2.72060200 & -3.64032200 & 6.35861500 \\
\hline $\mathrm{H}$ & -1.36284200 & -4.76001400 & 6.25158400 \\
\hline $\mathrm{H}$ & -1.11332200 & -3.16607800 & 6.99501000 \\
\hline $\mathrm{H}$ & 3.96054600 & -0.72390900 & 7.37107900 \\
\hline $\mathrm{H}$ & 11.87687700 & -5.16598100 & 0.04506800 \\
\hline $\mathrm{H}$ & 12.39994500 & -5.38090200 & -1.62909500 \\
\hline $\mathrm{H}$ & -3.36291400 & -0.15202500 & -1.46733500 \\
\hline $\mathrm{H}$ & -4.14592000 & 2.38481500 & -4.02766700 \\
\hline $\mathrm{H}$ & -2.65557200 & 2.19316300 & -3.05956200 \\
\hline $\mathrm{H}$ & -3.44958300 & 0.77237700 & -3.77601300 \\
\hline $\mathrm{H}$ & -4.62493100 & 2.51010600 & -1.54318400 \\
\hline $\mathrm{H}$ & 4.10417300 & -0.47036600 & -6.38109600 \\
\hline $\mathrm{H}$ & 4.82192900 & -1.98148000 & -7.00340400 \\
\hline $\mathrm{H}$ & 3.86397400 & -0.91175400 & -8.07161900 \\
\hline $\mathrm{H}$ & -2.15253900 & 5.38654400 & 2.62418800 \\
\hline $\mathrm{H}$ & -2.22023300 & 3.61105600 & 2.54513200 \\
\hline $\mathrm{H}$ & -0.65800800 & 4.43451300 & 2.76774100 \\
\hline $\mathrm{H}$ & -4.11339000 & -0.95938200 & -6.91933300 \\
\hline $\mathrm{H}$ & 1.45279200 & -1.07096800 & 0.37692600 \\
\hline $\mathrm{H}$ & 0.37684600 & -2.30444200 & 5.46451700 \\
\hline $\mathrm{H}$ & -6.14258400 & -2.49159600 & -1.91628700 \\
\hline
\end{tabular}
Total electronic Energy (HF): -9997.846017 